\title{
A Forty-Segment Molecular Synchrotron
}

By Peter C. Zieger ${ }^{1}$, Chris J. Eyles ${ }^{1}$, Sebastiaan Y. T. van de Meerakker², André J. A. van Roij ${ }^{2}$, Hendrick L. Bethlem ${ }^{3}$, and Gerard Meijer ${ }^{1,2, *}$

1 Fritz-Haber-Institut der Max-Planck-Gesellschaft, Faradayweg 4-6, 14195 Berlin, Germany

2 Radboud University Nijmegen, Institute for Molecules and Materials, Heyendaalseweg 135, 6525 AJ Nijmegen, The Netherlands

3 LaserLaB, Department of Physics and Astronomy, VU University Amsterdam, De Boelelaan 1081, $1081 \mathrm{HV}$ Amsterdam, The Netherlands

We acknowledge the design of the electronics by G. Heyne, V. Platschkowski, and T. Vetter and the software support by U. Hoppe. C. J. E. thanks the Alexander von Humboldt Foundation for a post-doctoral fellowship. S. Y. T. v. d. M acknowledges financial support from NWO via a VIDI grant. H. L. B. acknowledges financial support from NWO via a VIDI-grant, and from the ERC via a Starting Grant. G. M. acknowledges support from the ERC-2009-AdG under grant agreement 247142-MolChip.

(Received March 22, 2013; accepted in revised form July 18, 2013)

(Published online September 30, 2013)

\section{Cooling and Trapping of Molecules / Synchrotrons / Molecular Collisions}

We present a synchrotron for polar molecules consisting of 40 straight hexapoles arranged in a circle with a $50 \mathrm{~cm}$ diameter. The mechanical design and alignment procedure as well as the trigger scheme used to switch the voltages applied to the hexapoles are described in detail. The stability of the synchrotron is demonstrated by measurements in which multiple packets are stored for over $13 \mathrm{~s}$, during which they have completed over 1000 round trips and traveled a distance of over one mile. Furthermore, we demonstrate the simultaneous trapping of 26 packets; 13 revolving clock-wise and 13 counter clock-wise, that are injected into the synchrotron by two Stark decelerator beamlines. We discuss the opportunities for using the synchrotron as a low-energy collider.

\section{Introduction}

The ability to control the motion of neutral molecules offers exciting new possibilities for spectroscopic and collision studies [1-3]. One method by which this can be achieved is to exploit the interaction of polar molecules with electric fields. A polar molecule is a molecule whose charge is unequally distributed over the molecule, i.e., one side of the molecule is more positively charged and one side is more negatively charged. When such a molecule is placed in an electric field, the positive side of the

* Corresponding author. E-mail: meijer@fhi-berlin.mpg.de 
molecule experiences a force along the direction of the electric field, while the negative side of the molecule experiences a force in the opposite direction to the electric field. If the electric field is homogeneous, the two forces cancel and no net force acts on the molecule. However, if the electric field is inhomogeneous, the two forces no longer cancel. In this case, the net resultant force is given by: $\vec{F}=\mu_{\text {eff }} \vec{\nabla}|\vec{E}|$ with $\mu_{\text {eff }}$ being the effective dipole moment, which is given by the projection of the dipole moment onto the electric field vector. It is instructive to compare this formula with Coulomb's law, $\vec{F}=-q \vec{\nabla} V$, which describes the force exerted on a charged particle in a homogeneous electric field. The similarity between the two formulas suggests that we can manipulate polar molecules with electric field gradients in much the same way as charged particles are manipulated with voltage differences. There are complications, however. First, $\mu_{\mathrm{eff}}$ depends on the quantum state, and so it is crucial that the molecules remain in the same quantum state throughout the experiment [4-6]. Second, in general $\mu_{\text {eff }}$ is a function of the electric field. Third, in contrast to the electrostatic potential, the gradient of the electric field magnitude in free space is not equal to zero [7]. The second and third point introduce aggravating non-linearities in electric lenses [8,9]. Last but not least, the force experienced by polar molecules in inhomogeneous fields is typically some eight to ten orders of magnitude weaker than the forces that can be exerted on ions. Nevertheless, over the last decade it has been demonstrated that the motion of molecules in a molecular beam can be completely controlled using electric fields (see Ref. [10,11] and references therein).

This paper describes a synchrotron for polar molecules. It demonstrates the level of control that can nowadays be achieved over molecules; multiple packets of neutral molecules are made to revolve clockwise and anti-clockwise for more than 1000 revolutions in a tabletop storage ring, thereby traveling a distance of over one mile.

Confining molecules in a storage ring. Modern synchrotrons for charged particles are typically built out of many individual elements placed along a circular track [12,13]. These elements, separated by some free-flight distance, are designed for performing a specific task, e.g., injecting, steering, focusing, accelerating, bunching or extracting the particles. A scheme for building a synchrotron for molecules in a similar fashion was presented by Nishimura et al. [14], however, the acceptance of such a design is seriously hindered by the non-linearities of the forces experienced by the molecules. We have opted for a rather different approach. In our synchrotron, only one element a hexapole - is used. It can be shown that the electric field around the average radial position of the molecules (the equilibrium orbit, see Sect. 2.3), can be decomposed into a constant, a linear and a quadratic term [15]. The linear term delivers the necessary force for steering the beam, the quadratic term focuses the molecule transversally, while by modulating the constant term the molecules are focused in the longitudinal direction. To inject and extract the molecules the voltages on the electrodes are adjusted to obtain a homogeneous electric field of relatively small magnitude. Although, this was not obvious to us from the outset, the analysis presented in Ref. [15] suggests that a hexapole geometry, approximated by circular rods, is in fact the most favorable geometry in terms of acceptance, ease of construction and required electronics.

In the experiments presented in this paper, we deal exclusively with polar molecules in low-field seeking states, i. e., molecules that have their dipole moment oriented anti- 


\section{A Storage Ring for Neutral Molecules}

A Molecular Synchrotron
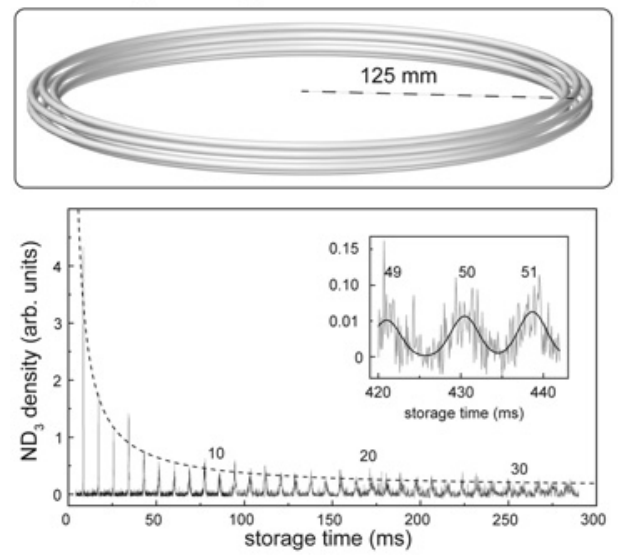
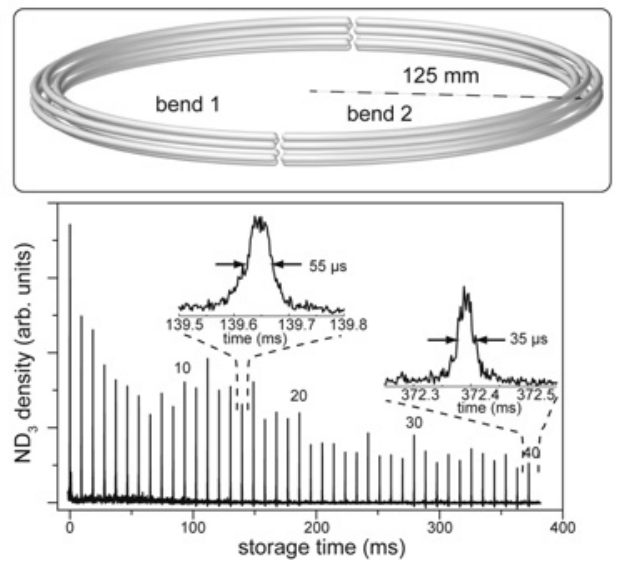

Fig. 1. The two predecessors of the present molecular synchrotron. The top left panel shows a photo realistic image of the first generation storage ring for neutral molecules, consisting of a hexapole guide bent into a circle of radius $125 \mathrm{~mm}$. Below the ring, the $\mathrm{ND}_{3}$ intensity at the detection point is shown as a function of storage time (reproduced from [23]). To guide the eye, the dashed line indicates a $1 / t$ decay. The inset shows the ammonia intensity for the $49^{\text {th }}$ to the $51^{\text {st }}$ round trips. It is seen that after 50 round trips the packet has filled the entire ring.

The top right panel shows the second generation molecular storage ring: the prototype molecular synchrotron, consisting of two hexapoles bent into a semi circle, separated by $2 \mathrm{~mm}$ wide gaps. The measurement under the ring shows the $\mathrm{ND}_{3}$ intensity as a function of storage time (reproduced from [24]). Here, due to the applied switching scheme, the molecular packet is confined as a tight bunch in an effective three dimensional potential well.

parallel to the electric field. Schemes for synchrotrons that store molecules in high-field seeking states based on alternating gradient focusing have been proposed by Nishimura et al. [16] and by de Nijs and Bethlem [17], while a storage ring composed of a toroidal wire was proposed by Loesch and Scheel [18]. Magnetic storage rings have been used to store low energy neutrons [19] and atoms [20-22].

The molecular storage ring described in this paper is the third generation storage ring for polar molecules built in our laboratory. The first and second generation storage rings are shown schematically in Fig. 1. The top left panel of Fig. 1 shows a storage ring that is obtained by bending a linear hexapole focuser into the shape of a torus, as proposed by Katz [25] and demonstrated by F. M. H. Crompvoets and coworkers [26]. In this prototype storage ring, the molecules are transversely confined but are essentially free along the longitudinal direction, i. e., along the circle. The lower left panel of Fig. 1 shows a measurement of the $\mathrm{ND}_{3}$ density at the detection point in the ring as a function of storage time. For long storage times the spatial width of the packet gradually increases until, eventually, the molecules fill the entire ring.

After the successful implementation of this first storage ring, C.E. Heiner and coworkers demonstrated a sectioned storage ring consisting of two hexapoles bent into a semicircle with a radius of $125 \mathrm{~mm}$, separated by a $2 \mathrm{~mm}$ gap [24]. By switching the electric field synchronously with the motion of the molecules, the electric fields in the gap are used to keep the molecular packet confined in the longitudinal direc- 
tion. A photo realistic image of this molecular synchrotron is shown in the top right panel of Fig. 1. The lower right panel of Fig. 1 shows a measurement of the $\mathrm{ND}_{3}$ density as a function of storage time at the detection point. By switching the electric fields, the width of the packet after 40 round trips was confined to only $3 \mathrm{~mm}$. In later measurements, Heiner and coworkers demonstrated that a packet of molecules was kept in a tight bunch even after completing 100 round trips, corresponding to a flight distance of $80 \mathrm{~m}$ [27]. The same group also showed the successful demonstration of the simultaneous confinement of two molecular packets, trailing each other by about $200 \mathrm{~mm}$ [24].

This paper describes the third generation of a molecular storage ring, consisting of forty straight hexapoles [28]. The increased number of segments has several advantages:

(i) the transverse acceptance of the synchrotron increases when the symmetry of the synchrotron increases. Variation of the confinement force as a function of the position in the ring leads to parametric amplification of the amplitude of the motion inside the ring at particular longitudinal velocities. These motional resonances are absent if the periodicity of the ring is much smaller than the typical length scale of the oscillatory motion. Note that motional resonances are also introduced by imperfections in the construction of the ring. However, if these imperfections are not too large the width and depth of these resonances are small and losses are kept to a minimum by carefully choosing the best operation conditions (see Sect. 3.4).

(ii) The depth of the longitudinal potential well scales with the inverse of the length of a single segment. Compared to the synchrotron that consisted of two half rings, the longitudinal well depth is expected to increase by a factor $\sqrt{10}$. Actually, in this paper a different bunching scheme is implemented that is a factor of three less efficient but much less demanding for the high voltage electronics. As a result the longitudinal well depth is about the same as that of Heiner et al.; however, we have come to realize that the previously used bunching scheme led to additional losses from the ring due to instabilities of the molecular trajectories and nonadiabatic transitions to nontrapped states. In the new bunching scheme these losses are absent.

(iii) The number of packets that can be trapped scales with the number of segments. In the experiments presented in this paper, we have trapped up to 13 packets of molecules revolving clock and counter-clock wise in the ring.

Although the synchrotron is useful for many studies, this paper will mainly focus on its possible use in collision studies. The ability to control the translational energy and energy spread of a molecular beam enables scattering experiments to be conducted that probe molecular interaction potentials in great detail. A holy grail of this research is to be able to observe resonances of the collision cross section as a function of collision energy. These resonances occur when kinetic energy is converted into rotational energy as a result of the anisotropy of the potential energy surface (PES) of the two collision partners; the width of the resonances is determined by the lifetime of this collision complex [29,30]. The velocity spread of a Stark-decelerated beam is in principle sufficiently small to resolve this resonant structure. Another motivation for research into cold collisions is the fact that at low temperatures the collision process becomes sensitive to externally applied electric or magnetic fields. This gives a handle to control and steer the outcome of a chemical reaction $[31,32]$. 
In 2006, Gilijamse et al. performed the first scattering experiment using a Starkdecelerated molecular beam [33]. In this experiment, $\mathrm{OH}$ radicals were scattered with a conventional beam of Xe atoms in a crossed molecular beam geometry. The total collision energy is changed by varying the velocity of the $\mathrm{OH}$ radicals, while the velocity of the rare gas beam is kept fixed. Subsequently, collisions of $\mathrm{OH}$ radicals with other rare gas atoms have been studied extensively, determining accurately the threshold behaviors of the state-to-state inelastic scattering cross sections $[34,35]$. Recently, experiments have been conducted using collisions between Stark-decelerated $\mathrm{OH}$ molecules and a state-selected beam of NO molecules [36]. Ultimately, one would like to study collisions between two velocity controlled molecular beams to resolve these resonances. In order to achieve this, one aims for molecular beams that are fully controlled, i.e., in a single quantum state and with a controlled absolute velocity and velocity spread. Thus far, the densities achieved have been insufficient for such experiments.

The obvious advantage of a ring structure for collision studies is that a stored packet of molecules revolving around the ring will repeatedly encounter counter-propagating packets. By storing many packets over an extended time, the sensitivity with which collisions can be detected increases by orders of magnitude compared to an experiment where the collision partners encounter each other only once. In Sect. 4 measurements will be presented demonstrating the simultaneous trapping of 13 packets revolving in both directions. A packet that has completed $64 \frac{1}{4}$ round trips (see Fig. 21) will have had 1670 encounters with packets revolving in the opposite direction.

Outline of this paper. In this paper we describe our experiments with a 40-segment synchrotron for polar molecules. It is based on the PhD-thesis of P. C. Zieger. Section 2 derives the theoretical background necessary to describe and understand the motion of the molecules inside the molecular synchrotron. Expressions are given for the trap frequencies that characterize the motion of the molecules inside the moving potential well. The equations presented in Sect. 2 are partially reproduced from the PhD theses of F. M. H. Crompvoets [23] and C. E. Heiner [37]. Section 3 presents experiments that demonstrate that full control is achieved over the motion of the packet of state-selected molecules in a molecular storage ring. A decelerated molecular packet is confined for over $13 \mathrm{~s}$; at this time it has traveled over a mile and has passed through 41000 gaps. The distance of one mile testifies to the intrinsic stability of the molecular trajectories. A number of other measurements that characterize the synchrotron are also presented in this section. Finally, Sect. 4 shows the simultaneous confinement of counter-propagating packets. The currently achievable densities of the trapped molecules are not yet sufficient to detect the desired bi-molecular collisions.

\section{Confining neutral molecules in a synchrotron}

Figure 2 shows a schematic of the molecular synchrotron. It consists of forty straight hexapoles which are placed on a circle with a diameter of half a meter $\left(R_{\text {ring }}=250 \mathrm{~mm}\right)$. Neighboring hexapoles are separated by a $2 \mathrm{~mm}$ gap. Each hexapole segment has an in- 


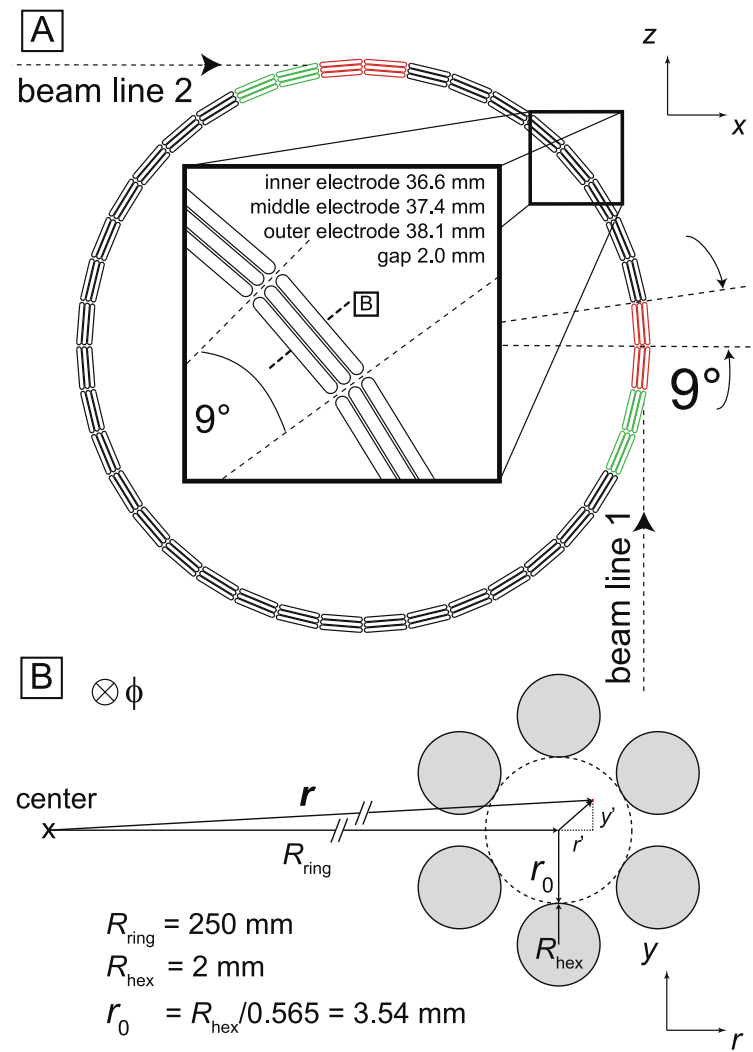

Fig. 2. A schematic of the molecular synchrotron. The top panel (A) shows a top view of the forty straight hexapoles aligned onto a circle with a radius of $250 \mathrm{~mm}$. The angle between adjacent hexapoles is $9^{\circ}$. Two Stark decelerator beam lines inject packets of molecules tangentially at two positions in the synchrotron. When a new packet is injected, the two hexapoles shown in green and the two hexapoles shown in red are switched off at the appropriate times. The hexapoles in red are also used as extraction field for the laser-produced ions. The inset shows a detailed view of a hexapole segment. To ensure a constant gap of $2 \mathrm{~mm}$ the electrode rods have different lengths. The lower panel (B) shows the cross section inside a hexapole segment. Following Anderson [38], the ratio between the rod radius $R_{\text {hex }}$ and the inner radius of the hexapole $r_{0}$ is chosen to be 0.565 .

ner radius of $r_{0}=3.54 \mathrm{~mm}$. The electrodes have a radius of $R_{\mathrm{hex}}=2 \mathrm{~mm}$, such that the ratio of $R_{\text {hex }} / r_{0}$ is 0.565 , which yields a good approximation to an ideal hexapole field using cylindrical rods [38]. In order to maintain a constant gap of $2 \mathrm{~mm}$ between two neighboring hexapole segments, electrodes in each hexapole segment are longer on the outside and shorter on the inside. The position of the molecule, $r$, is described with the three coordinates $\phi, r^{\prime}$ and $y^{\prime} . \phi$ is the angle of the molecule in the $x$-z-plane relative to the detection point of the first beam line (between the two red hexapole segments of the first beam line on the top panel of Fig. 2). $r^{\prime}$ is the radial coordinate and $y^{\prime}$ the vertical coordinate with respective to the center of the hexapole. More details on the experimental setup are given in Sect. 3. In this section the equations of motion for polar molecules inside the molecular synchrotron are derived. 


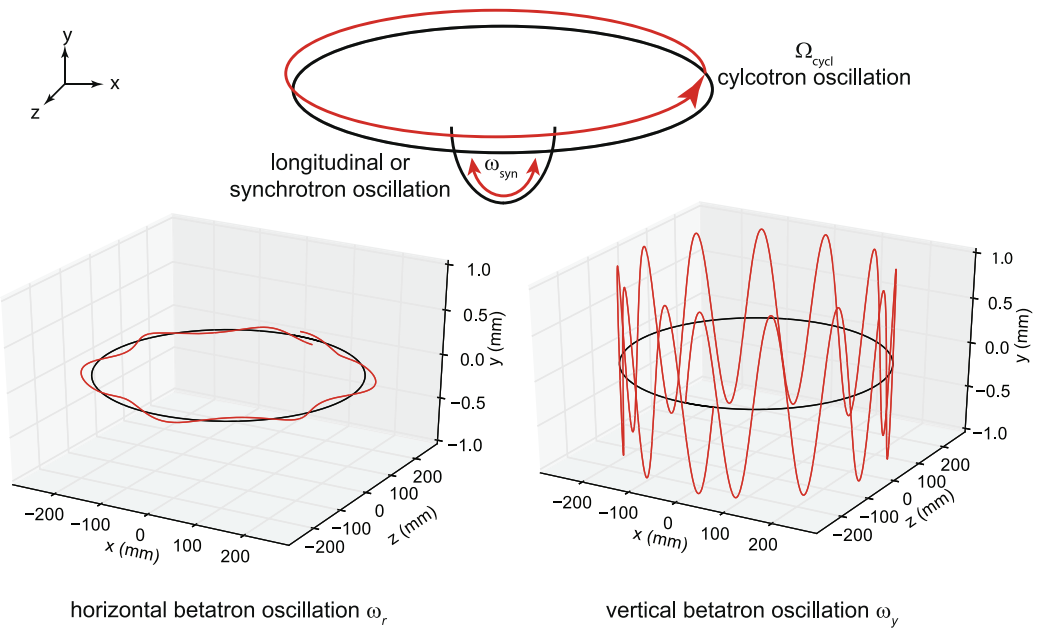

Fig. 3. A simplified schematic of the different oscillation types that a molecule performes in the molecular synchrotron. The black curve represents the trajectory of the synchronous molecule (equilibrium orbit). The red curve is a trajectory of a non-synchronous molecule performing various oscillations. The upper part shows the angular cyclotron frequency $\Omega_{\text {cycl }}$, which is $2 \pi$ over the time that the synchronous molecules needs to complete one full round trip. The synchrotron frequency $\omega_{\text {syn }}$ is the oscillation frequency in the longitudinal direction. The lower panel shows the two transverse oscillations. On the left is the horizontal or radial betatron oscillation $\omega_{r}$ and on the right the vertical betatron oscillation $\omega_{y}$.

\subsection{Characteristic frequencies}

Figure 3 shows a simplified schematic of the different types of oscillations performed by molecules inside the molecular synchrotron. The motion is best described by four characteristic frequencies. In keeping with the nomenclature used for charged particle accelerators, we refer to these as the cyclotron, synchrotron, and (horizontal and vertical) betatron frequencies. The black curves represent the trajectory of a hypothetical molecule that makes a closed orbit in the synchrotron. We call this molecule the synchronous molecule, while we call its orbit the equilibrium orbit. By definition, the equilibrium orbit is at the position where the centrifugal force and the force created by the hexapoles cancel each other [39]. The cyclotron frequency is the inverse of the time that the molecule needs to complete one full round trip (round-trip time). The cyclotron angular frequency is $\Omega_{\text {cycl }}=2 \pi / t_{\mathrm{rt}}$. In our experiments $\Omega_{\text {cycl }} / 2 \pi$ is typically $78 \mathrm{~Hz}$. While the cyclotron frequency describes the velocity of the traveling potential well, molecules will oscillate in all three directions within this moving frame. In the longitudinal direction the molecules oscillate at the synchrotron frequency, $\omega_{\mathrm{syn}}$. In the transverse directions the molecules oscillate with the horizontal and vertical betatron frequency, $\omega_{r}$ (lower left panel) and $\omega_{y}$ (lower right panel), respectively. The characteristic frequencies depend on the confining voltages that are applied on the electrodes as well as on the velocity of the traveling potential well.

The characteristic frequencies are derived in the following sections and are summarized in Table 1 for a fixed velocity and confining voltage difference. In 
Table 1. Characteristic frequencies of ammonia molecules in a forty-segment molecular synchrotron. The longitudinal velocity of the stored packet is $124.3 \mathrm{~m} / \mathrm{s}$ while a voltage difference of $6 \mathrm{kV}$ is used. The first column shows the theoretical values that are obtained in Sections 2.3 and 2.5. The middle column lists the experimentally determined frequencies presented in this paper while the last column shows the results obtained via the resonant excitation technique [40].

\begin{tabular}{lccc}
\hline type & $\begin{array}{c}\text { fheoretical } \\
\text { (this section) }\end{array}$ & $\begin{array}{c}\text { frequencies } \\
\text { experimental } \\
\text { (Section 3) }\end{array}$ & $\begin{array}{c}\text { experimental } \\
\text { (from Ref. [40]) }\end{array}$ \\
\hline $\begin{array}{l}\text { cyclotron frequency } \\
\begin{array}{l}\Omega_{\text {cycl }} / 2 \pi \\
\text { synchrotron frequency }\end{array}\end{array}$ & $78 \mathrm{~Hz}$ & $78 \mathrm{~Hz}$ & $78 \mathrm{~Hz}$ \\
$\begin{array}{l}\omega_{\text {syn }} / 2 \pi \\
\text { horizontal betatron frequency } \\
\omega_{r} / 2 \pi \\
\text { vertical betatron frequency }\end{array}$ & $58 \mathrm{~Hz}$ & $68(2) \mathrm{Hz}$ & $64(2) \mathrm{Hz}$ \\
$\omega_{y} / 2 \pi$ & $879 \mathrm{~Hz}$ & $891(11) \mathrm{Hz}$ & $890(1) \mathrm{Hz}$ \\
\hline
\end{tabular}

Sect. 3, both betatron frequencies are determined from the observed stop bands in the synchrotron, while the synchrotron frequency is determined from expansion measurements. These frequencies are listed in the second column of Table 1. In Zieger et al. [40], the characteristic frequencies are derived from measurements in which the amplitude, or the duration, of the HV-pulses that are applied to the hexapoles is modulated.

\subsection{The Stark effect of the ammonia molecule}

The ammonia molecule. The ammonia molecule is a classic textbook example of a pyramid-shaped symmetric-top molecule [41]. The three hydrogen atoms (or in the case of its deuterated form $\mathrm{ND}_{3}$ the three deuterium atoms) build the ground plane of the pyramid while the nitrogen atom sits at its apex. The rotational structure of $\mathrm{NH}_{3}$ and $\mathrm{ND}_{3}$ is best described by two principal quantum numbers $J$ and $K$, which are the total angular momentum and its projection onto the molecular axis, respectively. The umbrella motion in ammonia is strongly hindered due to the interaction of the hydrogen atoms with the nitrogen that leads to a barrier. Due to quantum mechanical tunneling through this barrier each rotational level in the ground state of ammonia is split into a symmetric and an antisymmetric component. For the $|J, K\rangle=|1,1\rangle$ state, used in the experiments described in this paper, this splitting is $0.79 \mathrm{~cm}^{-1}$ [42] and $0.053 \mathrm{~cm}^{-1}$ [43] for $\mathrm{NH}_{3}$ and $\mathrm{ND}_{3}$, respectively.

The Stark effect in ammonia. The charges of ammonia isotopologues are unevenly distributed over the molecule, leading to a body-fixed electric dipole moment. In the ground state of $\mathrm{NH}_{3}$ this dipole moment is equal to 1.47 Debye [44], while it is 1.50 Debye in the ground state of $\mathrm{ND}_{3}$ [45]. The Stark shift is given by the eigenvalues 


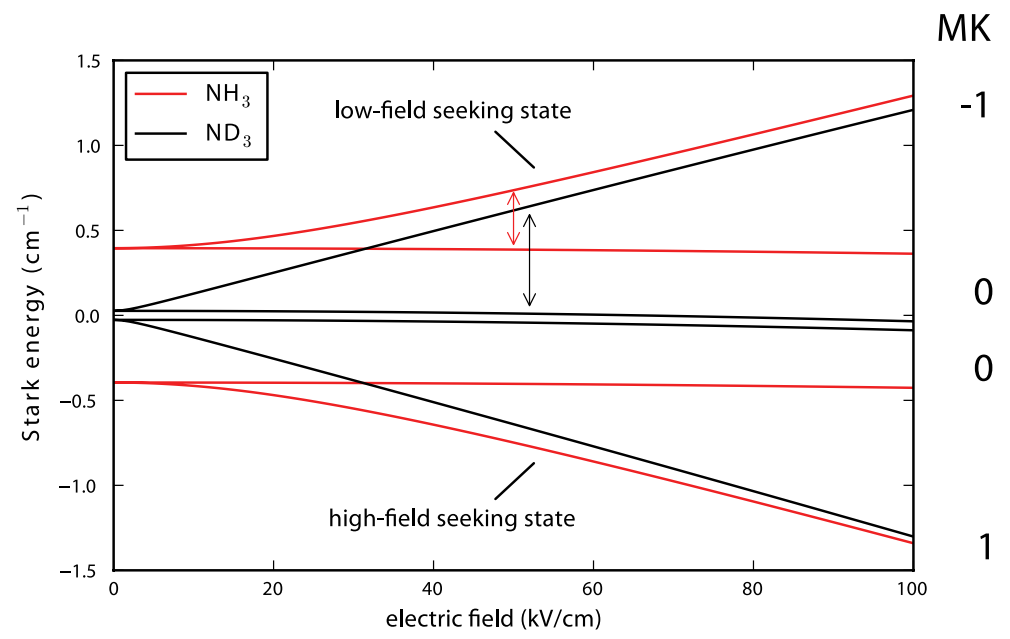

Fig. 4. The Stark energy of the $|J, K\rangle=|1,1\rangle$ state of $\mathrm{NH}_{3}$ and $\mathrm{ND}_{3}$ as a function of the electric field. The red and the black arrows indicate the change in Stark energy at an electric field strength of $50 \mathrm{kV} / \mathrm{cm} \mathrm{for}$ ammonia and its deuterated isotopologue, respectively.

of $H_{0}+H_{\text {Stark }}$, where $H_{0}$ is the field-free hamiltonian and $H_{\text {Stark }}$ is given by

$$
\widehat{H}_{\text {Stark }}=-\boldsymbol{\mu} \cdot \boldsymbol{E} .
$$

It is convenient to use rigid rotor wave functions $|J, K, M, p\rangle$ as the eigenfunctions of the rotational state, where $M$ is the projection of the total angular momentum on the space-fixed axis, $\boldsymbol{\mu}$ is the electric dipole moment and $\boldsymbol{E}$ the electric field, while $p$ indicates the parity. The upper and lower inversion doublet of ammonia have opposite parity, thus they are coupled by the Stark Hamiltonian in first order. Assuming that the energy difference between different rotational states is large, the resulting Stark energy of the interacting inversion doublet levels is to a first approximation given by [45]

$$
E_{\mathrm{Stark}}= \pm \sqrt{\left(\frac{W_{\mathrm{inv}}}{2}\right)^{2}+\left(\mu E \frac{M K}{J(J+1)}\right)^{2}} \mp\left(\frac{W_{\mathrm{inv}}}{2}\right),
$$

with $W_{\text {inv }}$ being the above mentioned inversion splitting of ammonia.

Figure 4 shows the energy of the ground state of para-ammonia as a function of the electric field. The product of $M$ and $K$, which in the following will be referred to as $M K$, is 0 or +1 in the symmetric state, and 0 or -1 in the antisymmetric state. In the presence of an electric field some energy levels shift with increasing electric field. For $M K=+1$ the Stark energy decreases with increasing electric field strength, while for $M K=-1$ the Stark energy increases. Molecules in the $M K=-1$ state are called low-field-seekers because they lower their energy by moving to regions with the lowest field. Conversely, molecules in the $M K=+1$ state are high-field-seekers. To a first order approximation, the $M K=0$ states are not affected by the electric field.

The main reason why the experiments in this paper are performed with $\mathrm{ND}_{3}$ and not with $\mathrm{NH}_{3}$ is due to the different Stark effect of both isotopologues. As indicated 
by the red and black arrows in Fig. 4, the relative change in Stark energy of a lowfield-seeking molecule in a given electric field is larger for $\mathrm{ND}_{3}$ than for $\mathrm{NH}_{3}$. In other words, a deuterated ammonia molecule which moves into an inhomogeneous electric field will gain more Stark energy than its non deuterated isotopologue. It will therefore lose more kinetic energy which is advantageous for the process of Stark deceleration (see [46]). A second benefit of $\mathrm{ND}_{3}$ is the linearity of the Stark shift. Compared to $\mathrm{NH}_{3}, \mathrm{ND}_{3}$ has a small inversion splitting and is thus more sensitive to low electric fields $(0-50 \mathrm{kV} / \mathrm{cm})$.

\subsection{Transversal confinement}

Force, equilibrium radius and equilibrium height. In a perfect hexapole that is bent into a torus - such as the first storage ring for neutral molecules that was built in our lab [39] - the centrifugal force is balanced by the radial component of the hexapole field. The centrifugal force can be written as

$$
F_{\text {centri }}=\frac{m v_{\phi}^{2}}{R_{\text {ring }}+r^{\prime}} .
$$

Here, $r^{\prime}$ is the radial position inside the hexapole, $v_{\phi}$ is the longitudinal velocity and $m$ the mass of the molecule inside the synchrotron. The force in the radial direction on a deuterated ammonia molecule in the low-field seeking $|J, M K\rangle=|1,-1\rangle$ state inside a hexapole is given by

$$
F_{\text {hex }}=-\frac{k r^{\prime}}{\sqrt{1+\left(\frac{W_{\text {inv }}}{k\left(r^{\prime 2}+y^{\prime 2}\right)}\right)^{2}}} .
$$

Here, $W_{\text {inv }}$ is the inversion splitting of this state and $k$ is the harmonic force constant which is itself determined by the voltage difference $V_{0}$ that is applied to the hexapole rods, the inner radius $r_{0}$ of the hexapole as well as by the quantum state of the molecule

$$
k=\mu \frac{3 V_{0}}{2 r_{0}^{3}} \frac{|M K|}{J(J+1)} .
$$

Combining both equations leads to

$$
\frac{m v_{\phi}^{2}}{R_{\text {ring }}+r^{\prime}}=\frac{k r^{\prime}}{\sqrt{1+\left(\frac{W_{\text {inv }}}{k\left(r^{\prime 2}+y^{\prime 2}\right)}\right)^{2}}} .
$$

The particular radius where the two forces cancel each other is referred to as the equilibrium radius, $r_{\text {equi }}^{\prime}$. The inversion splitting in deuterated ammonia is small compared to $k r_{\text {equi }}^{\prime 2}$ and Eq. (6) can be rewritten as

$$
r_{\text {equi }}^{\prime}=\frac{R_{\text {ring }}}{2}\left[\sqrt{1+\left(\frac{2 v_{\phi}}{R_{\text {ring }} \omega}\right)^{2}}-1\right] \approx \frac{v_{\phi}^{2}}{R_{\text {ring }} \omega^{2}}
$$



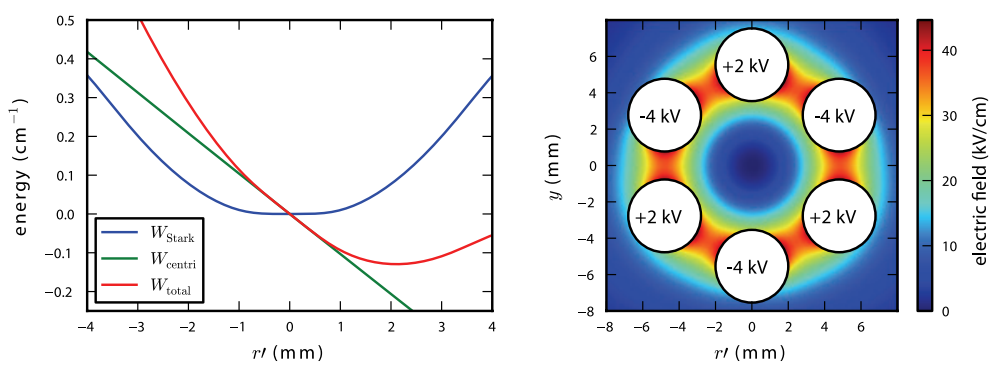

Fig. 5. Left panel: The Stark energy $W_{\text {Stark }}$ for an $\mathrm{ND}_{3}$ molecule in the low-field seeking $|J, M K\rangle=$ $|1,-1\rangle$ state as a function of displacement from the hexapole center (blue curve) $\left(y^{\prime}=0 \mathrm{~mm}\right)$. With a forward velocity of $124.3 \mathrm{~m} / \mathrm{s}$ the molecule finds itself inside the molecular synchrotron in a centrifugal pseudo-potential $W_{\text {centri }}$ (green curve). The sum of the two energies is the effective potential energy $W_{\text {total }}$ (red curve) that the molecules experience transversally $\left(W_{\text {total }}=W_{\text {stark }}+W_{\text {centri }}\right)$. Right panel: cross section of one hexapole segment showing the electric field.

with $\omega$, the betatron frequency given by

$$
\omega=\sqrt{\frac{k}{m}} .
$$

The last approximation in Eq. (7) is valid if $2 v_{\phi} \ll R_{\text {ring }} \omega$. In the vertical direction the force of gravity needs to be compensated,

$$
-m g=\frac{k y^{\prime}}{\sqrt{1+\left(\frac{W_{\text {inv }}}{k\left(r^{\prime 2}{ }_{\text {equi }}+y^{\prime 2}\right)}\right)^{2}}} .
$$

In analogy to the equilibrium radius, we will refer to the particular height where the two forces cancel each other as the equilibrium height, $y_{\text {equi }}^{\prime}$. If we again neglect the inversion splitting we find

$$
y_{\text {equi }}^{\prime} \approx-\frac{g}{\omega^{2}} .
$$

When a voltage difference of $6 \mathrm{kV}$ is applied between the rods of the hexapole, the betatron frequency, $\omega /(2 \pi)$ for deuterated ammonia in the low-field seeking $|J, K\rangle=|1,1\rangle$ state is equal to $880 \mathrm{~Hz}$ (i.e., in a perfect hexapole ring, when the inversion splitting is neglected, the radial and vertical betatron frequencies are equal). At a forward velocity of $124.3 \mathrm{~m} / \mathrm{s}$, we find from Eqs. (7) and (10) an equilibrium radius of $r_{\text {equi }}^{\prime}=2.0 \mathrm{~mm}$ and an equilibrium height of $y_{\text {equi }}^{\prime}=-0.32 \mu \mathrm{m}$. The small gravitational distortion justifies the neglect of the effect of gravity hereafter.

The above derivation assumed a perfect hexapole field. The right panel of Fig. 5 shows the electric field for a hexapole composed of cylindrical rods. As a result of using cylindrical rods, the electric field close to the electrodes is seen to deviate from the ideal field. If this effect, as well as the inversion splitting, are taken into account the equilibrium radius becomes $2.1 \mathrm{~mm}$. 
Potential energy and betatron frequency. The effective potential energy $W_{\text {total }}$ that a molecule experiences in the molecular synchrotron is the sum of the Stark energy $W_{\text {Stark }}$ and the pseudo-potential energy of the centrifugal force, $W_{\text {centri }}$. By integrating the left side of Eq. (6) over $\mathrm{d} r^{\prime}$, the pseudo-potential can be written as

$$
W_{\text {centri }}=-m v_{\phi}^{2} \ln \left|1+\frac{r^{\prime}}{R_{\text {ring }}}\right| .
$$

Neglecting the inversion splitting, the resulting potential is

$$
W_{\text {total }}=\frac{1}{2} k r^{\prime 2}-m v_{\phi}^{2} \ln \left|1+\frac{r^{\prime}}{R_{\text {ring }}}\right| .
$$

The left panel of Fig. 5 shows the Stark energy (blue curve), centrifugal psuedopotential (green curve) and the sum of these two potentials (red curve) as a function of the radial position in the ring. These calculations use the numerically calculated electric field and include the inversion splitting. The forward velocity is taken as $124.3 \mathrm{~m} / \mathrm{s}$. With increasing velocity, the tilt of the pseudo-potential becomes steeper and the effective total potential energy becomes shallower while the minimum of the total potential energy (the equilibrium radius) is displaced further from the geometrical center of the hexapole. In Sect. 3.4, measurements are presented where the velocity of the molecules is varied. In these measurements, the attractive potential well is kept at constant depth and position by adjusting the voltages applied to the hexapole electrodes in accordance with the velocity of the molecular packet.

The radial betatron frequency can be found by taking the second derivative of the effective potential energy with respect to the radial position at the equilibrium radius. For a longitudinal velocity of $124.3 \mathrm{~m} / \mathrm{s}$ we find $\omega_{r} / 2 \pi=879 \mathrm{~Hz}$. Similarly, the vertical betatron frequency is found to be $\omega_{y} / 2 \pi=860 \mathrm{~Hz}$.

Phase-space acceptance. An important quantity that characterizes the synchrotron is the phase-space acceptance, A. The phase-space acceptance is defined as the volume in position and velocity space that can be occupied by the molecules, that is, a number with units of $(\mathrm{m} \cdot \mathrm{m} / \mathrm{s})^{3}$. Depending on the context the acceptance may also refer to the boundary that encloses the phase-space volume occupied by the molecules [11]. Assuming a linear restoring force, the acceptance of a ring is

$$
A_{r}=\pi r_{\max } v_{r, \max }
$$

with $r_{\max }$ and $v_{r, \max }$ being the distance to the equilibrium radius and the radial velocity a molecule may have while still being confined in the ring. Assuming an ideal hexapole and neglecting the inversion splitting, the maximum distance to the equilibrium radius is

$$
r_{\max }=r_{0}-r_{\text {equi }}^{\prime} \text {. }
$$

The corresponding maximum transverse velocity $v_{\mathrm{r}}$ is

$$
v_{r, \max }=\omega r_{\max } \approx \omega\left(r_{0}-\frac{v_{\phi}^{2}}{R_{\text {ring }} \omega^{2}}\right) .
$$




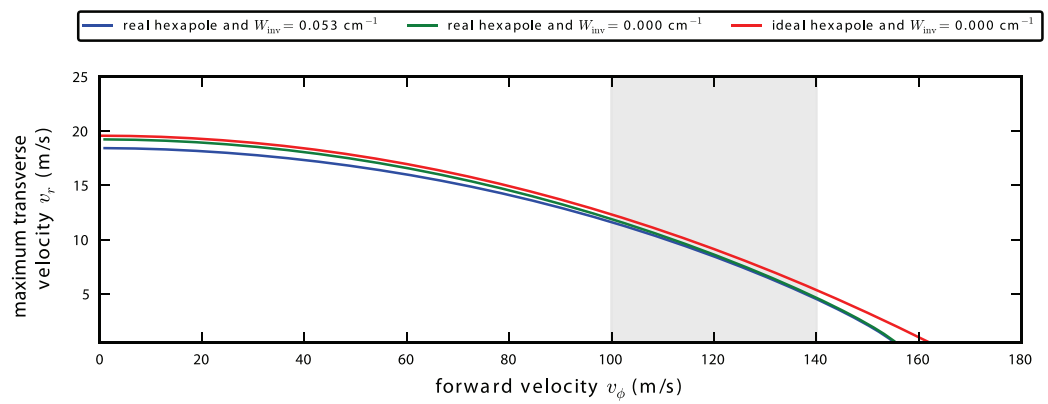

Fig. 6. The figure shows the maximum transverse velocity a molecule can have and still be confined transversally in the synchrotron for three different situations: The blue curve corresponds to calculations in which the inversion splitting is taken into account and the hexapole field is calculated with a finite-element program [47]. For the green curve the inversion splitting is set to zero. The red curve corresponds to calculations that use the electric field for an ideal hexapole and neglects the inversion splitting $(\omega / 2 \pi)=$ $879 \mathrm{~Hz}$ ). The shaded region shows the velocity range normally used in the molecular synchrotron.

With a forward velocity of $124.3 \mathrm{~m} / \mathrm{s}$ and a voltage difference of $6 \mathrm{kV}$ a maximum transverse velocity of $8.4 \mathrm{~m} / \mathrm{s}$ is accepted in the synchrotron. The radial acceptance is $38 \mathrm{~mm} \cdot \mathrm{m} / \mathrm{s}$. Figure 6 shows the maximum transverse velocity $v_{r, \max }$ as a function of forward velocity $v_{\phi}$. The red curve assumes a perfect hexapole and is calculated using Eq. (15) at a constant frequency of $879 \mathrm{~Hz}$. The green and blue curve show the maximum velocity obtained from the effective potential using the numerically calculated electric field and setting the inversion splitting at zero (green curve) or at its correct value (blue curve).

By combining Eq. (13) to (15) we find

$$
A_{r}=\pi \omega\left(r_{0}-\frac{v_{\mathrm{f}}^{2}}{R_{\text {ring }} \omega^{2}}\right)^{2} .
$$

In a similar fashion, the vertical acceptance of the ring is given by

$$
A_{y}=\pi y_{\max } v_{y, \max }=\pi \omega y_{\max }^{2}=\pi \omega\left(r_{0}^{2}-r_{\text {equi }}^{\prime 2}\right)=\pi \omega\left(r_{0}^{2}-\left(\frac{v_{\mathrm{f}}^{2}}{R_{\text {ring }} \omega^{2}}\right)^{2}\right) .
$$

For a longitudinal velocity of $124.3 \mathrm{~m} / \mathrm{s}$ the maximum vertical velocity is $15.3 \mathrm{~m} / \mathrm{s}$ and the acceptance is $120 \mathrm{~mm} \cdot \mathrm{m} / \mathrm{s}$.

\subsection{Transverse stability in a segmented ring}

All equations derived in the previous sections are valid for a hexapole that is bent into a perfect circle. In our synchrotron, we use forty straight hexapoles to approximate a circle. In this section, we analyse how this changes the acceptance and motivate why we have decided to use forty hexapoles and not more or less. ${ }^{1}$ To understand this number

\footnotetext{
${ }^{1}$ This question is addressed in the thesis of C.E. Heiner [37]. Her analysis is partly reproduced here.
} 
we need to look at the occurrence of so-called motional resonances. Imperfections in a circular structure lead to transverse loss as the molecules experience a periodic deviation in the restoring force that leads to parametric amplification of the amplitude of the oscillatory motion of the molecules in the ring. This phenomenon is well known in particle accelerators [13] and has already been investigated in the previous molecular synchrotron [48]. Motional resonances occur if the number of transverse oscillations per segment is either a half integer or whole integer value. The radial and vertical betatron tune $v_{r}$ and $v_{y}$ are defined as the number of transverse oscillations per segment:

$$
v_{r}=\frac{\omega_{r}}{n_{\mathrm{seg}} \omega_{\mathrm{cycl}}} \quad \text { and } \quad v_{y}=\frac{\omega_{y}}{n_{\mathrm{seg}} \omega_{\mathrm{cycl}}},
$$

with $n_{\text {seg }}$ the number of (identical) segments that the ring is composed of. Primarily, $\omega_{\text {cycl }}$ depends on the velocity of the molecular packet and $\omega_{r, y}$ on the applied voltage. As a result of motional resonances the trajectories are unstable for certain combinations of the velocity and applied voltage. In keeping with the terminology used in particle physics the unstable regions are called 'stop bands' [13]. Under the assumption that the molecule experiences a linear Stark effect inside a perfect hexapole field, the betatron tune in the radial and vertical direction is given by

$$
v_{r, y}=\frac{\sqrt{k_{r, y} / m}}{n_{\text {seg }} 2 \pi v_{f} /\left(R_{\text {ring }}+r_{\text {equi }}\right)}=\frac{R_{\text {ring }}+r_{\text {equi }}}{n_{\text {seg }} v_{\mathrm{f}}} \sqrt{\frac{3 \mu_{\text {eff }} V}{m r_{0}^{3}}} .
$$

Forty segments make almost a good circle. The upper panel in Fig. 7 shows the acceptance that results from simulating the trajectories of 20000 molecules through a molecular synchrotron consisting of different number of linear hexapoles as a function of the forward velocity. The radius of the molecular synchrotron is $0.25 \mathrm{~m}$ and the hexapoles have an inner radius $r_{0}$ of $3.54 \mathrm{~mm}$. The force on a molecule inside a hexapole segment $n$ is assumed to be perfectly linear with a fixed frequency of $879 \mathrm{~Hz}$ and a force constant of

$$
k=m \omega^{2} .
$$

The position of a molecule inside the molecular synchrotron in the $x$ - $z$-plane is described with the angle $\phi$ which necessitates a coordinate transformation to the laboratory frame (see Fig. 2)

$$
\sin \phi=\frac{z}{r}=\frac{z}{\sqrt{x^{2}+z^{2}}} .
$$

A molecule is in the straight hexapole segment $n$, if the following condition is fulfilled

$$
\frac{n-1 / 2}{n_{\mathrm{seg}}}<\frac{\phi}{2 \pi}<\frac{n+1 / 2}{n_{\mathrm{seg}}},
$$

This model assumes that there are no gaps between individual straight parts. A molecule is considered to be lost if its distance to the geometrical center of a hexapole becomes 

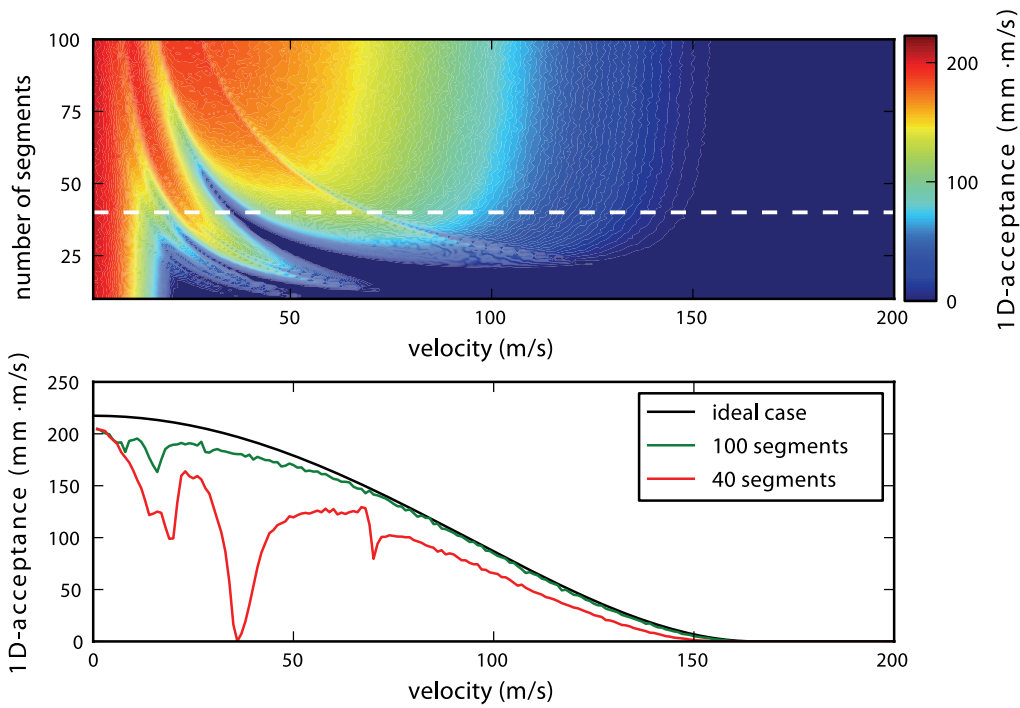

Fig. 7. Upper panel: The numerically calculated phase-space acceptance in the horizontal plane of a molecular synchrotron as a function of forward velocity and number of straight segments. The radial frequency is set to $\omega_{r} /(2 \pi)=879 \mathrm{~Hz}$. The horizontal dashed white line corresponds to a synchrotron consisting of forty hexapoles. Lower panel: The phase-space acceptance for a synchrotron consisting of either 40 hexapoles (red curve) or 100 hexapoles (green curve). The black curve shows the acceptance for an ideal hexapole torus using Eq. (16).

larger than the inner radius of the hexapole

$$
r_{0}<\sqrt{y^{\prime 2}+r^{\prime 2}}
$$

The fraction of molecules that remain inside the molecular synchrotron after $200 \mathrm{~ms}$ is multiplied by the initial phase-space distribution to obtain the phase-space acceptance $A$. The initial position spread of the random packet is chosen to be $8 \mathrm{~mm}$ and the initial velocity spread is $40 \mathrm{~m} / \mathrm{s}$ which is larger than the acceptance for all settings. Each point in the upper panel of Fig. 7 represents the one dimensional phase-space acceptance at a specific number of segments and velocity. It is obvious that when the ring is composed out of more straight segments, the motional resonances are shifted to lower velocities and become less dominant. In other words, the higher the symmetry of the synchrotron (number of straight segments) the fewer motional resonances occur. The black curve in the lower panel of Fig. 7 shows the acceptance for a perfect ring symmetry and is calculated using Eqs. (16) and (7). The green curve shows the simulated acceptance for 100 segments. Only for velocities below $50 \mathrm{~m} / \mathrm{s}$ does it differ from the ideal case. The red curve shows the transverse stability for 40 segments. From this graph it was decided to build the current forty segment molecular synchrotron as a compromise between acceptance and construction effort. The calculation in Fig. 7 assumes that every hexapole is perfectly aligned and the same voltage difference is applied to each hexapole segment. In reality, the alignment of each hexapole segment with respect to the centerline 


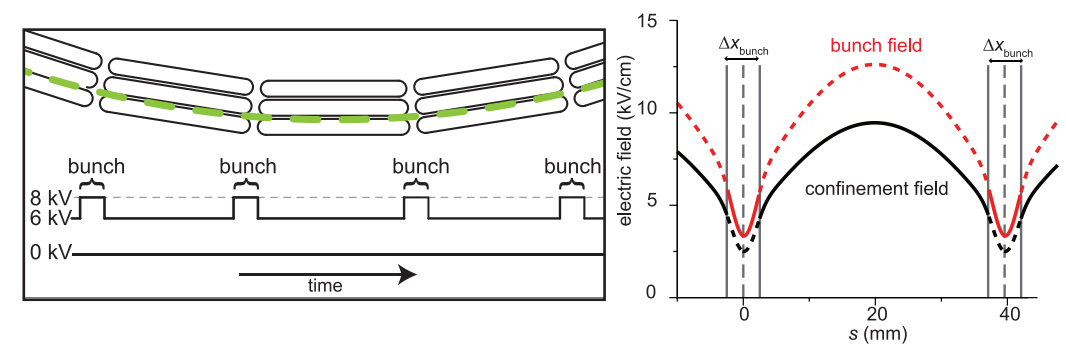

Fig. 8. Left panel: Scheme of the time dependence of the voltage difference between adjacent hexapole electrodes during operation of the synchrotron. By temporarily switching to a higher voltage difference whenever the packet passes through a gap, ammonia molecules are kept in a tight bunch while revolving. The green dashed curve indicates the equilibrium orbit at a forward velocity of $124.3 \mathrm{~m} / \mathrm{s}$. Right panel: Electric field strength (both for confinement and bunching) as a function of position along the equilibrium orbit. The origin of the horizontal axis $s$ is located at the midpoint between two hexapole segments.

will be slightly different and the voltage difference applied will not be identical. These effects again lead to a deviation of the restoring force and hence to regions of instability. Note that the periodicity of these deviation is $1 / \Omega_{\text {cycl }}$ rather than $1 / n_{\text {seg }} \Omega_{\text {cycl }}$. Experimental measurements of the stability of the synchrotron are presented in Sect. 3.4.

\subsection{Longitudinal confinement}

The previous section showed that molecules are confined transversally (in the $y$-and $r$-direction) by applying a constant voltage on the forty hexapoles that are placed on a circle. This section discusses how to confine a molecular packet in a tight bunch along the longitudinal direction. The left side of Fig. 8 shows a schematic of the timedependent switching scheme. The electric field is switched synchronously with the revolving packet in the synchrotron between the confinement field and the so-called bunching field (the electric field of the bunching field is 4/3 times stronger than of the confinement field). The time variation of the voltage in sync with the motion of the molecules is the eponym of the molecular synchrotron. On the right side of Fig. 8 the electric fields for the confinement and the bunching configuration are shown as a function of position along the equilibrium orbit (dashed green line on the left panel) in black and red, respectively. For a time duration $\Delta t_{\text {bunch }}$ the electric field is switched to the bunching configuration every time the synchronous molecule is in the gap region. This time is translated into an effective bunching length $\Delta x_{\text {bunch }}$, which is determined by the final velocity $v_{\mathrm{f}}$ of the synchronous molecule that is injected by the Stark decelerator beam line

$$
\Delta x_{\text {bunch }}=v_{\mathrm{f}} \Delta t_{\text {bunch }} .
$$

In Fig. 9 the operation principle of the longitudinal bunching scheme is described in more detail. It is similar to that of the buncher in the decelerator beam line (described in [49]). The upper panel shows the potential energy of the low field seeking component of the $|J, M K\rangle=|1,-1\rangle$ state as a function of longitudinal position along the equilibrium orbit for the two switching configurations. The confinement field corresponds 

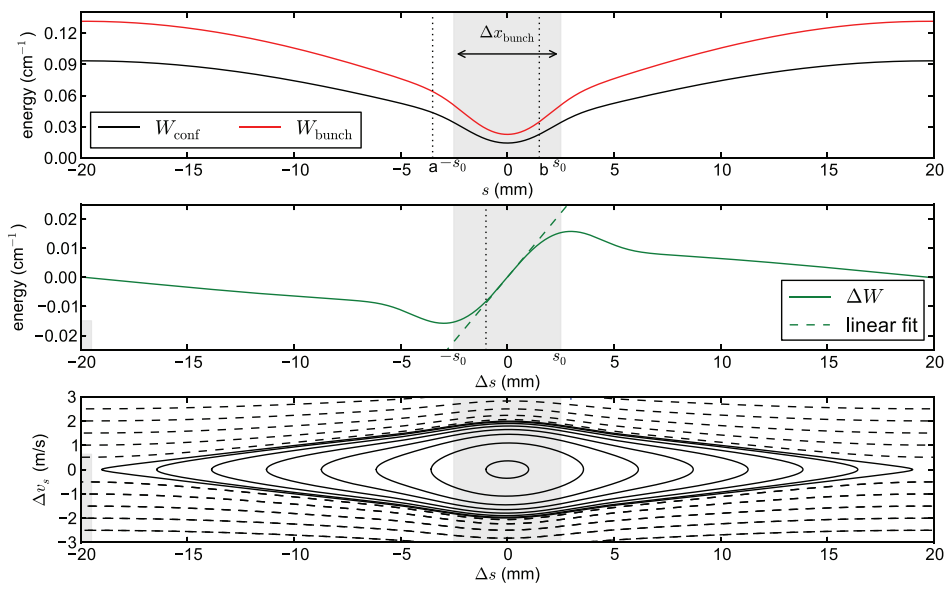

Fig. 9. The upper panel shows the potential energy of an ammonia molecule in the $|J, M K\rangle=|1,-1\rangle$ state as a function of longitudinal position along the equilibrium orbit. The potential of the confinement and bunching field is shown in black and red, respectively. The origin of the horizontal axis is located at the midpoint between two hexapole segments. In the gray shaded region the synchronous molecule experiences the bunching field. The effective length $\Delta x_{\text {bunch }}$ is $5 \mathrm{~mm}$ and is symmetric around the gap region. The middle panel shows the change in potential energy as a function of position. A molecule that is behind the synchronous molecule (left dotted line) experiences a negative change in potential energy and will be pushed towards the synchronous molecule. The restoring force for a molecule is approximately linear for a length of $5.0 \mathrm{~mm}$. Near the synchronous molecule the slope of $\Delta W$ is $8.8 \mathrm{~cm}^{-1} / \mathrm{m}$. The lower panel shows the phase-space diagram in the longitudinal direction. The solid lines correspond to molecules that are stably bunched while trajectories in dashed lines are not stably bunched.

to the black and the bunching field to the red curve. Let us first consider the motion of the synchronous molecule along the equilibrium orbit in the confinement configuration. If the electric field is not switched, it will lose potential energy and gain kinetic energy as it moves along the electric fringe fields in the gap region. After passing the middle of the gap, the synchronous molecule will regain all the potential energy it lost and will have the same kinetic energy as before it entered the gap. If now the electric field is switched from the confinement field to the bunching field for an effective length $\Delta x_{\text {bunch }}$ symmetrically around the synchronous molecule (gray shaded region), the synchronous molecule will gain and lose a larger amount of kinetic energy. After the switching process the velocity of the synchronous molecule still remains unchanged.

For non-synchronous molecules, the change in potential energy $\Delta W$ depends on the longitudinal position $s$ of the molecule along the equilibrium orbit as the field is switched to the bunching configuration.

$$
\begin{aligned}
\Delta W(s)= & \left(W_{\text {bunch, } \mathrm{a}}(s)-W_{\text {conf }, \mathrm{a}}(s)\right) \\
& -\left(W_{\text {bunch, } \mathrm{b}}\left(s+\Delta x_{\text {bunch }}\right)-W_{\text {conf }, \mathrm{b}}\left(s+\Delta x_{\text {bunch }}\right)\right) .
\end{aligned}
$$

We will denote the position of the synchronous molecule when the field is switched to the bunching and confining configuration by $\pm s_{0}$, respectively. Furthermore, we introduce the $\Delta s$, the position of a non-synchronous molecule with respect to the syn- 
chronous molecule when the field is switched to the bunching configuration, $s=-s_{0}+$ $\Delta s$. The change in energy can now be written as

$$
\begin{aligned}
\Delta W(s)= & \left(W_{\text {bunch,a }}\left(-s_{0}+\Delta s\right)-W_{\text {conf,a }}\left(-s_{0}+\Delta s\right)\right) \\
& -\left(W_{\text {bunch, }}\left(s_{0}+\Delta s\right)-W_{\text {conf, }}\left(s_{0}+\Delta s\right)\right) .
\end{aligned}
$$

$\Delta W$ is shown in the middle panel of Fig. 9 for a synchronous velocity of $124.3 \mathrm{~m} / \mathrm{s}$, and an effective bunching length $\Delta x_{\text {bunch }}=5 \mathrm{~mm}$. The horizontal axis shows the position of a molecule with respect to the synchronous molecule. The synchronous molecule $(\Delta s=0)$ will not change its kinetic energy during the switching process. A molecule that is, e.g., - in position - behind the synchronous molecule (dotted line) experiences a stronger acceleration than deceleration in the gap and will gain kinetic energy. Consequently, it will be pushed towards the synchronous molecule. Conversely, a molecule that is in front of the synchronous molecule will be decelerated and pushed back towards the synchronous molecule.

The average longitudinal force $\bar{F}$ that a molecule experiences while passing a hexapole segment of length $\Delta L$ can be expressed as

$$
\bar{F}=-\frac{\Delta W(s)}{\Delta L} .
$$

For molecules close to the synchronous molecule ( $\Delta s$ is small), the restoring force is linear and the force can be written as

$$
\bar{F}=-\frac{\Delta W^{\prime}(s) \Delta s}{\Delta L},
$$

with $\Delta W^{\prime}(s)$ being the slope of the potential difference. $\Delta W^{\prime}(s) / \Delta L$ is the harmonic force constant $k$. The corresponding longitudinal angular frequency is

$$
\omega_{\mathrm{syn}}=\sqrt{\frac{\Delta W^{\prime}}{m \Delta L} .}
$$

The green dashed line in the middle panel of Fig. 9 shows a linear fit of the potential difference $\Delta W$. From this fit we find $\Delta W^{\prime}=8.8 \mathrm{~cm}^{-1} / \mathrm{m}$. Furthermore, the length of an hexapole segment $\Delta L=2 \pi\left(R_{\text {ring }}+r_{\text {equi }}\right) / 40$ is $39.6 \mathrm{~mm}$. Thus we find $\omega_{\text {syn }} /(2 \pi)=$ $58 \mathrm{~Hz}$.

The lower panel of Fig. 9 shows a number of trajectories in phase-space calculated using the longitudinal potential (middle panel) and the resulting average force. In the harmonic part of the well (inner solid circle) the synchrotron frequency $\omega_{\text {syn }} /(2 \pi)$ is $60 \mathrm{~Hz}$.

\subsection{Numerical simulation}

In order to get a better understanding of the synchrotron we have performed numerical simulations of the trajectories of molecules inside the molecular synchrotron. The benefit of these simulations is that experimental measurements can be reproduced and confirmed. The simulations also enable us to obtain quantities that cannot be directly 
measured, and give us insight into the effects of misalignments and coupling of the different motions in the synchrotron. Unfortunately, the coupling makes it difficult to test the program for errors in the code; due to the Coriolis coupling it is impossible to perform 1D calculations. In the next section some of the experimental measurements are compared to simulations. This is not done for all measurements because a calculation in which 100000 molecules revolve in the molecular synchrotron for several seconds is time consuming and requires a large amount of computer resources (CPU and RAM). For example, in the simulation of Fig. 17 (right panel) the trajectories of 100000 molecules are calculated for 100000 different voltage-velocity configurations with a Supercomputer using 100 CPUs. It took $41 / 2$ days to finish the calculation. This makes it impractical to optimize the different initial conditions.

The symmetry of the molecular synchrotron implies that the electric potential of only one segment needs to be calculated precisely. The electric potentials for the other segments are determined via a transformation of coordinates from the initial segment. The electric potential of the first segment is calculated as a grid with Simion [47] using 20 grid points $/ \mathrm{mm}$. The force that a molecule experiences in this potential grid is determined by the second derivative of the potential field. In order to simulate a molecular beam that is injected into the molecular synchrotron from a Stark decelerator beam line, a packet of molecules with a Gaussian position and velocity spread is generated. The trajectory of each molecule is traced until a certain number of round trips have been completed inside the synchrotron. If a molecule hits an electrode during its passage, or if it is outside of the hexapole, the calculation of that specific molecule is stopped and a new trajectory with new initial conditions is started. The number of detected molecules at the end of the simulation corresponds to the relative density of molecules in the experiment. A small additional random force is added to the confinement force in each segment in order to reproduce the effect of small misalignments in the synchrotron (see Sect. 3).

The left three panels of Fig. 10 show the results of a simulation of the trajectories of 100000 molecules that make a 100 round trips within the synchrotron. The top, middle and bottom panels show the phase-space distribution in the radial, vertical and longitudinal directions, respectively. The initial position and velocity distributions in the three dimensions are $2.5 \mathrm{~mm}$ and $2.5 \mathrm{~m} / \mathrm{s}$ (FWHM), respectively and are plotted as gray points in each panel. The initial conditions should be an adequate approximation to simulate the output of the two deceleration beam lines. The solid and dashed lines show some trajectories in phase space resulting from the models presented in Sects. 2.3 and 2.5. The phase-space distribution after 100 round trips is shown in green, red and blue for the radial, vertical and longitudinal direction, respectively. It is seen that the initial phase-space distribution is smeared out in all six dimensions and fills the complete acceptance volume. The final distribution in the radial direction (green points) is displaced with respect to the model. The equilibrium radius is larger than that predicted by the model ( $r^{\prime}$ equi is 2.5 instead of $2.1 \mathrm{~mm}$ ). This is attributed to the fact that the model assumes a hexapole that is bent into a perfect circle, while the numerical simulations takes into account that the ring is formed by straight hexapoles. The figures shows the radial distribution of the molecules in the middle of one segment. It would be more appropriate (but also more complicated) to use a time-averaged contour plot. 

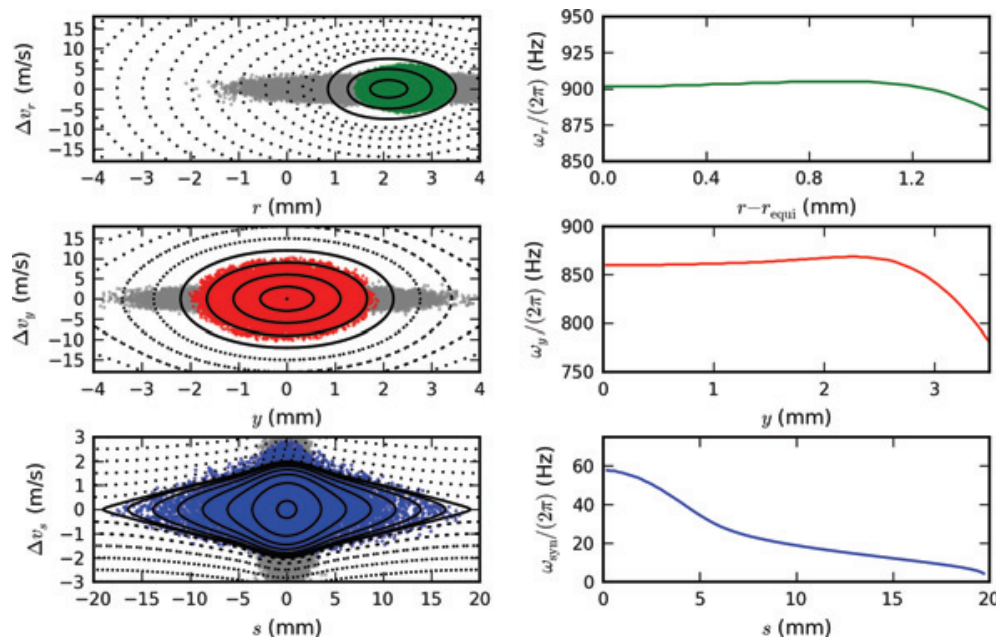

Fig. 10. For the radial, vertical and longitudinal directions, a phase-space plot is shown in the left top, middle and bottom panel, respectively. In this simulation the trajectories of 100000 molecules are calculated while they make up to 100 round trips in the synchrotron. The gray points indicate the initial molecular phase-space distribution; the colored points show the final simulated phase-space distribution. The right top, middle and bottom panel show the characteristic frequency as function of distance from the synchronous molecules from analytic models for the radial, vertical and longitudinal direction, respectively.

In the vertical direction the distribution (red points) does not fill the entire phasespace acceptance. The size of the vertical phase-space acceptance is determined by the amplitude of the vertical motion and its equilibrium orbit (see loss condition in Eq. (23) and substitute $r^{\prime}$ with $r_{\text {equi }}^{\prime}$. The vertical phase-space acceptance inherits the inaccuracy in $r_{\text {equi }}^{\prime}$. The longitudinal distribution (blue points) fills the entire phase-space acceptance. The slight asymmetry in the longitudinal velocity is not yet understood.

The characteristic frequency for the radial, vertical and longitudinal directions as a function of distance to the synchronous particle is shown in the three right panels of Fig. 10. The characteristic frequency for the synchronous molecule is $902 \mathrm{~Hz}, 860 \mathrm{~Hz}$ and $58 \mathrm{~Hz}$ for the radial, vertical and longitudinal frequency, respectively. The values are similar or equal to the values listed in Table 1.

\section{Experimental characterization of a forty segment synchrotron}

In this section we will present the lay-out of the synchrotron. We give a detailed description of the mechanical design and alignment procedure as well as the high voltage switches and associated electronics. Packets of ammonia molecules with a tunable velocity and adjustable velocity spread are injected clockwise and counter clockwise into the synchrotron by two molecular beam decelerators. The first beam line was already used in the experiments of Heiner and co-workers and is described in detail in ref. [49]. The second beam line is newly built [15]. It is similar to the first beam line, differing only in the number of stages that can be used for decelerating and bunching the 


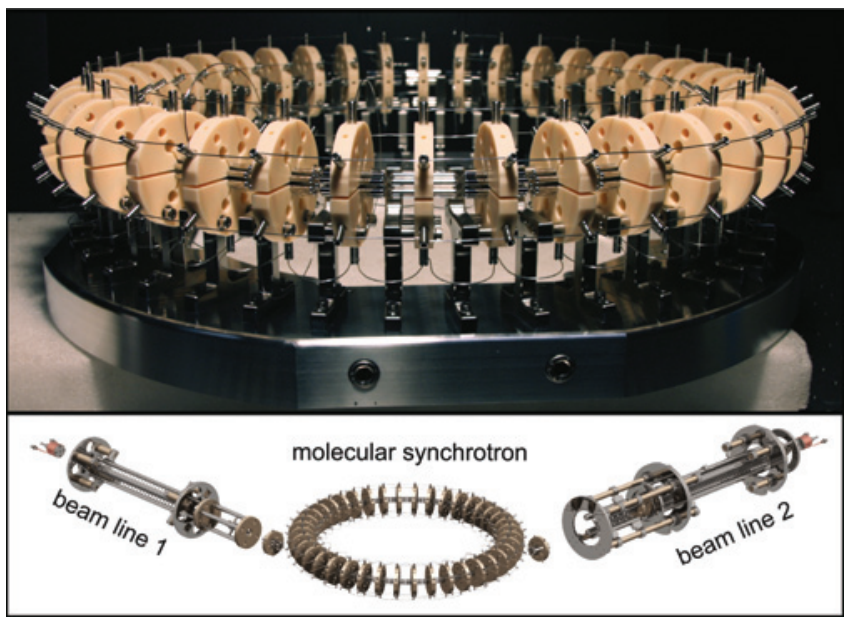

Fig. 11. Upper panel: A photograph of the molecular synchrotron consisting of 40 straight hexapoles separated by a $2 \mathrm{~mm}$ gap. Lower panel: A photo-realistic image of the molecular synchrotron together with the two Stark decelerator beam lines.

molecules. Furthermore, instead of a general valve, a Jordan valve is used. The two beam lines give similar signals.

\subsection{Experimental setup}

A photograph of the assembled molecular synchrotron is shown in Fig. 11. The diameter of the ring is $500 \mathrm{~mm}$. Each hexapole segment consists of six cylindrical highly polished electrodes with a rod diameter of $4 \mathrm{~mm}$, rounded off at each end. To guarantee a constant gap of $2 \mathrm{~mm}$ between neighboring hexapole segments, the two inner electrodes are $36.6 \mathrm{~mm}$, the two middle electrodes are $37.4 \mathrm{~mm}$ and the two outer electrodes are $38.1 \mathrm{~mm}$ long (see Fig. 2). The electrodes are mounted on an aluminum oxide disc that also serves as insulator. High voltage tests show that discharges between electrodes tend to occur along the shortest pathway across the ceramic surface. A slit in the ceramic between two neighboring electrodes minimizes the chance of a discharge, by maximizing the path length along the ceramic. The ceramic disc together with the electrodes is mounted with three screws onto a stainless steel holder. Using a special alignment tool, the center of the hexapole is aligned with respect to the holder with a precision of less than a tenth of a millimeter. These holders are then placed with set pins onto an aluminum base plate under an angle of $9^{\circ}$ with respect to each other. The resulting precision between neighboring hexapole segments is on the order of one tenth of a millimeter.

The forty segments are internally connected in three groups: (i) 32 hexapoles (sketched in black in Fig. 2) are switched continuously between the confinement and bunching configuration. (ii) 4 hexapoles (sketched in green in Fig. 2), 2 hexapoles for each beam line, are turned off to allow the injection of new molecular packet into the molecular synchrotron. (iii) 4 hexapole (sketched in red in Fig. 2), 2 hexapoles for each 
beam line, are turned off to allow the injection of new molecular packet into the molecular synchrotron and are switched to appropriate voltages to extract ions for detection.

\subsubsection{High voltage switches}

Seven high voltage power supplies are used for the two beam lines and the molecular synchrotron (manufactured by FUG (HCN 700-12500) and Spellman (SL15*1200)). The necessary thirty high voltage switches (four for the first beam line, five for the second beam line and twenty one for the molecular synchrotron) are manufactured by Behlke Electronic GmbH (HTS-151-03-GSM) and configured by the electronic workshop of the Fritz-Haber-Institut. The bias voltage is generated by eight power supplies (Delta Electronika BV (ES 0300-0.45)). The positive electrodes require two switches in series to switch between the confinement, the bunching and the detection/incoupling configuration. The negative electrodes switch between the confinement and detection/incoupling configuration. The lower voltage input varies depending on which electrode in which segment the switch is connected to. For the molecular synchrotron in total 21 high voltage switches are used: (i) 3 switches are used for the main section; one switch for the negative electrodes and two switches for the positive electrodes, which switch between ground and $-4 \mathrm{kV}$ and between ground, $+2 \mathrm{kV}$ and $+4 \mathrm{kV}$, respectively. (ii) 6 switches are used for the injection section; for each beamline one switch for the negative electrodes and two switches for the positive electrodes, which switch between ground and $-4 \mathrm{kV}$ and between ground, $+2 \mathrm{kV}$ and $+4 \mathrm{kV}$, respectively. (iii) 12 switches are use for the detection section; For each beam line, two switches are used to switch the voltage applied to the top electrodes between $-300 \mathrm{~V}$ (detection), $+2 \mathrm{kV}$ (confinement) and $+4 \mathrm{kV}$ (bunching), one switch is used for switching the voltage applied to the middle top electrodes between $-130 \mathrm{~V}$ (detection) and $-4 \mathrm{kV}$ (confinement), two switches are used to switch the voltage applied to the middle bottom electrodes between $+130 \mathrm{~V}$ (detection), $+2 \mathrm{kV}$ (confinement) and $+4 \mathrm{kV}$ (bunching), and finally one switch is used to switch the voltage applied to the bottom electrodes between $+300 \mathrm{~V}$ (detection) and $-4 \mathrm{kV}$ (confinement).

\subsubsection{Detecting ammonia molecules with $(2+1)$ REMPI}

The packets that are injected by two Stark decelerator beam lines are detected simultaneously using the same laser beam. The deuterated ammonia molecules are detected via $(2+1)$ resonantly enhanced multi-photon ionization (REMPI) [50] using pulsed laser light around $317 \mathrm{~nm}$. To detect both beam lines simultaneously, the laser beam is reflected and refocused $(f=125 \mathrm{~mm}$ ) inside the synchrotron into the detection point for beam line 2 . The ionized molecules are extracted by applying an appropriate voltage on the detection hexapoles such that ions drift upwards between two hexapole segments (right side of Fig. 12). In a $50 \mathrm{~cm}$ long time-of-flight tube, kept on a potential of $-1 \mathrm{kV}$, ions with different mass drift apart such that the deuterated ammonia molecules are separated from ions of different mass [51]. These ions are detected on a microchannel plate (MCP). The measured ion signal is proportional to the ammonia density in the laser focus. 

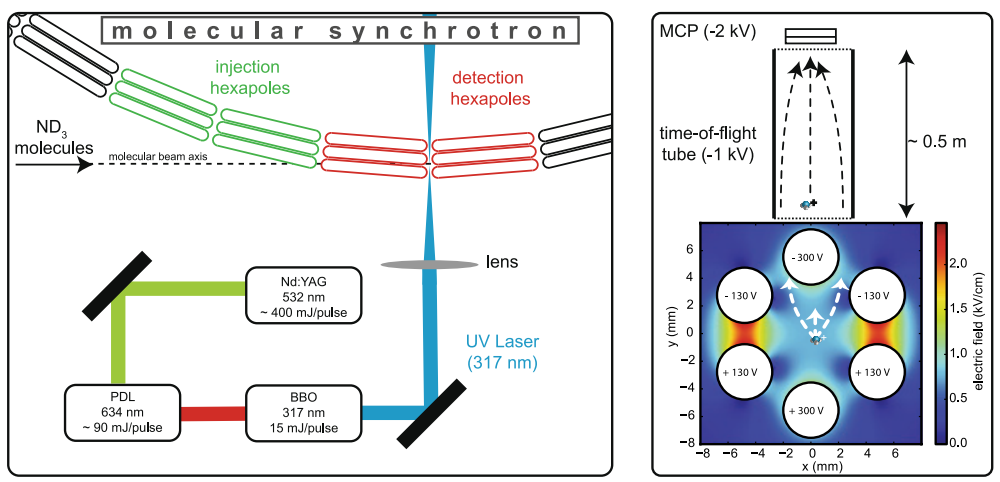

Fig. 12. Detection Scheme. Left: A high power UV laser pulse $(15 \mathrm{~mJ} /$ pulse $-5 \mathrm{~ns}$ duration $)$ is generated by pumping a dye laser with a Nd:YAG laser at $532 \mathrm{~nm}$ and doubling the resulting frequency with a BBO crystal. The laser beam is focused in between two hexapoles of the synchrotron using a lens with a focal length of $500 \mathrm{~mm}$. The molecules are ionized by a $(2+1)$ REMPI scheme. In this way molecules can be detected at the injection point each time they have completed an integer number of round trips.

Right: During the REMPI process extraction voltages are applied on the detection hexapoles. Positive ions experience an upward force and enter the time-of-flight tube. In the tube different masses drift apart and can be measured selectively by a microchannel plate (MCP) detector.

\subsubsection{Number of simultaneously stored packets}

Theoretically a forty segment molecular synchrotron with forty gaps allows the simultaneous confinement of eighty molecular packets: forty packets traveling clockwise, and forty traveling anti-clockwise. The molecular synchrotron is loaded such that each stored packet is in a gap region when a new packet is injected. In practice the spacing between successive packets must be at least two segments as the detection and injection hexapoles are connected in pairs of two. When a new packet is injected, the injection and detection hexapoles need to be turned off. With a spacing of one segment, a packet in the same section of the synchrotron will therefore be lost. If only one beam line is used up to 20 packets can be stored simultaneously. If both beam lines are used the upper limit of stored packets from one beam line is determined by the injection and detection technique of the other beam line. The procedure of injection and detection inside the synchrotron is synchronized such that the stored packets are not affected. With counter-propagating packets in the ring this is best realized if all packets are located simultaneously inside the main part of the first section consisting of the 24 hexapoles (see Fig. 2). The maximum number of simultaneously stored packets is

$$
n_{\text {packets }}=2 \cdot\left(\left\lfloor\frac{24}{n_{\text {spacing }}}\right\rfloor+1\right),
$$

where $n_{\text {spacing }}$ is the spacing between successive packets in units of segments. With a spacing of two hexapole segments, up to 26 packets can be confined simultaneously. In this paper the spacing between successive packets is generally two or three segments. To illustrate the advantage of confining multiple packets simultaneously, let us consider a molecular packet with a velocity of $124.3 \mathrm{~m} / \mathrm{s}$. To measure a single packet after, $e$. g., 
1000 round trips and averaging each point 100 times takes $100 \times 12.7 \mathrm{~s}=21.2 \mathrm{~min}$. With 13 simultaneous revolving packets, each measuring point takes only $1.8 \mathrm{~min}$.

\subsubsection{Continuous and pulsed trigger scheme}

The detection laser and the deceleration beam lines require a repetition rate between 9 and $11 \mathrm{~Hz}[52,53]$. The time delay between the injection of successive packets, which is referred to $t_{100 \mathrm{~ms}}$, needs to be found such that

$$
0.09 \mathrm{~s}<t_{100 \mathrm{~ms}}<0.11 \mathrm{~s} .
$$

The time delay $t_{100 \mathrm{~ms}}$ after a new packet is injected $n_{\text {spacing }}$ segments in front of the most recently injected packet is

$$
t_{100 \mathrm{~ms}}=\left(n_{\mathrm{rt}} \cdot 40-n_{\mathrm{spacing}}\right) \cdot t_{\mathrm{seg}} .
$$

Here, $n_{\mathrm{rt}}$ is an integer number and $t_{\mathrm{seg}}$ the time that the synchronous molecule needs to pass one full segment. In this paper a packet makes 7 or 8 round trips before a new one is introduced. The desired trigger pulses are generated with arbitrary wave form generators (Agilent 33220A) and with modified delay clock generators (FHI electronic workshop). A $10 \mathrm{MHz}$ time reference clock is used such that all components have the same time basis (TCXO standard).

In the measurements presented in this paper, two different trigger schemes are used: a pulsed and a continuous mode. In both modes multiple molecular packets revolve inside the synchrotron for the same number of round trips before being detected. Depending on the experiment, one trigger scheme is advantageous over the other.

Pulsed trigger scheme. The upper panel of Fig. 13 shows a sketch of the pulsed trigger scheme. In this scheme, all timings are defined relative to a delay generator. This master clock determines when and how many molecular packets are decelerated and injected into the molecular synchrotron. Each packet is decelerated and injected using a specific time sequence. Between each sequence is a time delay of $t_{100 \mathrm{~ms}}$ such that the spacing between packets is exactly $n_{\text {spacing }}$ segments. Every packet stays in the synchrotron the desired number of round trips before laser pulses are sent in to detect each one of them. The time delay between each of the laser pulses is again $t_{100 \mathrm{~ms}}$. The advantage of this trigger scheme is that an arbitrarily high number of round trips can be measured. For a large number of packets the loading and detection time is rather long, making this scheme less suitable for collision studies.

Continuous trigger scheme. The continuous trigger scheme is shown in the lower panel of Fig. 13. In this scheme, the molecular synchrotron switches continuously. Packets of molecules are injected with a delay time of $t_{100 \mathrm{~ms}}$ such that the spacing between packets is exactly $n_{\text {spacing }}$ segments. The time at which the detection laser is triggered is relative to the injection time. This allows the injection of a molecular packet into an empty gap after a previous molecular packet is detected and has exited the synchrotron. With counter-propagating packets present a molecular packet with a velocity of $124.3 \mathrm{~m} / \mathrm{s}$ and a spacing of two segments can stay up to 103 round trips in the synchrotron before 


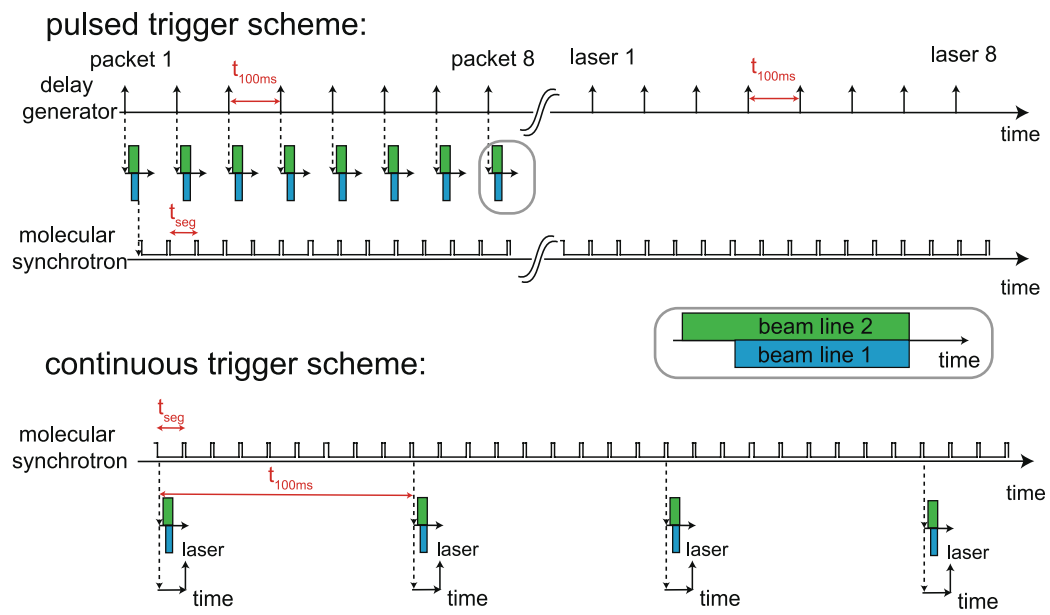

Fig. 13. The two trigger schemes of the molecular synchrotron. Subsequent packets are injected and detected with a time delay $t_{100 \mathrm{~ms}}$ of about $100 \mathrm{~ms}$ to assure that the repetition rate of the pulsed dye laser remains between 9 and $11 \mathrm{~Hz}$ (see Eq. 32). The upper panel shows the pulsed triggering scheme for the simultaneous confinement of eight packets. In this sketch we inject, $e$. g., eight molecular packets into the synchrotron, then wait until each packet completes the desired number of round trips before eight laser pulses are fired to detect them. The switching between confinement and bunching configuration starts after the injection of the first packet. The lower panel shows the continuous scheme, in which the molecular synchrotron is operated continuously. After the time delay of $t_{100 \mathrm{~ms}}$ a new molecular packet is injected. The time of the laser pulse is changed relative to the injection time. For reasons of clarity the horizontal axis of the molecular synchrotron in the upper and lower panel is exaggerated.

a new packet takes it place ( $n$ is 8 and $n_{\text {packets }}$ is 13 ). The maximum number of round trips is given by

$$
n_{\mathrm{rt}, \max }=n \cdot n_{\text {packets }}-1 \text {. }
$$

The disadvantage of the continuous scheme is the fixed maximum number of round trips. If, e. g., the depletion due to collisions is too small and it is desired to increase further the number of round trips it will be necessary to use the pulsed trigger scheme.

\subsection{Packets of neutral molecules revolving for over a mile}

Figure 14 shows the density of ammonia molecules as a function of time after injection into the synchrotron for a series of selected numbers of round trips. In this measurement thirteen packets are injected $\left(n_{\text {packets }}=13\right)$ using the pulsed trigger scheme, after which the loading is stopped. These packets trail each other by a distance of three hexapoles $\left(n_{\text {spacing }}=3\right.$ ). The first and the last packet are four hexapoles apart. The different peaks correspond to characteristic number of round trips, e. $g$., for the $640^{\text {th }}$ round trip the molecular packet is confined for $8.15 \mathrm{~s}$, corresponding to a flight length of one kilometer. Even after 1025 round trips, i.e., after the molecules have traveled a distance of over a mile and have passed through a gap 41000 times, their signal can be clearly recognized. The temporal width of $21 \mu$ s corresponds to a spatial distribution of $2.6 \mathrm{~mm}$. 


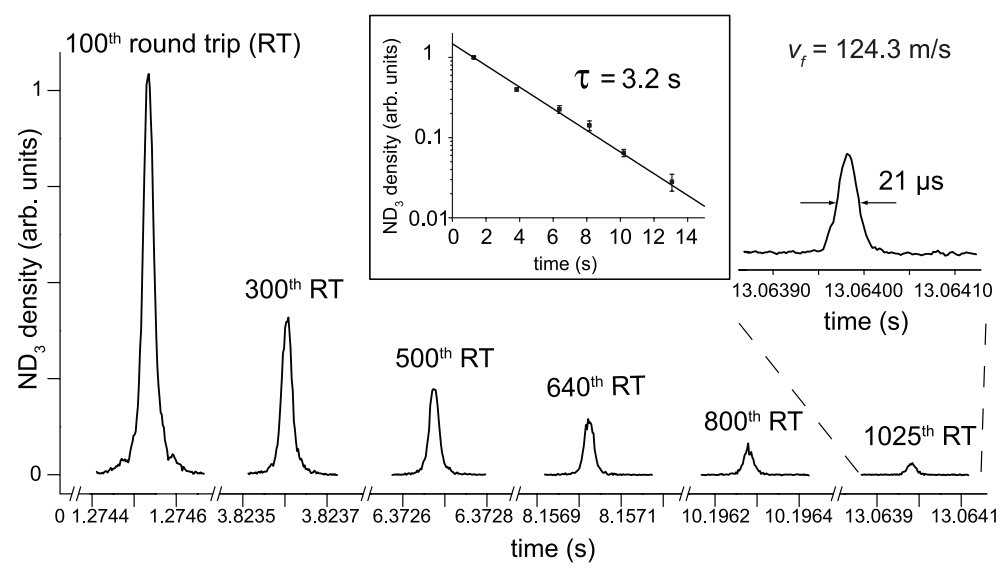

Fig. 14. Measurements of the density of $\mathrm{ND}_{3}$. molecules as a function of time (in seconds) for a selected number of round trips. The observed temporal width of $21 \mu$ s after 1025 round trips corresponds to a length of $2.6 \mathrm{~mm}$ after a flight distance of more than a mile. The inset shows the exponential decay of the signal with time. Figure is taken from reference [28].

This measurement explicitly demonstrates the stability of the trajectories of a molecular packet inside a molecular synchrotron.

The density of ammonia molecules is seen to decay exponentially with time at a rate of $0.31 \mathrm{~s}^{-1}$ (see inset of Fig. 14). This is the lowest decay rate that has been observed for neutral ammonia molecules in any trap to date. In all earlier electrostatic trapping experiments, 1/e-lifetimes of only a small fraction of a second were observed, probably limited by non-adiabatic transitions to non-trappable states near the trap center. This was only realized when substantially longer lifetimes of up to $1.9 \mathrm{~s}$ were measured in an electrostatic trap that had a non-zero electric field at the center [4]. With the present confinement and bunching scheme, the molecules are never close to a zero electric field in the synchrotron. In addition, although the magnitude of the electric field is changed, its direction is not. This prevents the occurrence of non-adiabatic transitions inside the hexapoles [5]. A major contribution to the observed loss rate is optical pumping of the ammonia molecules out of the $|J, M K\rangle=|1,-1\rangle$ level by blackbody radiation, calculated to occur at a rate of $0.14 \mathrm{~s}^{-1}$ at room-temperature [54]. The remaining loss-rate of $0.17 \mathrm{~s}^{-1}$ is well explained by collisions with background gas at the approximately $5 \times 10^{-9}$ mbar pressure in the vacuum chamber.

The longitudinal position spread can be determined from the time-of-flight (TOF) measurements presented in Fig. 14. Each of the TOF profiles is fitted with a Gaussian and from the fitted width the position spread is calculated. The spread is fairly constant over a trapping time of $13 \mathrm{~s}$ corresponding to 1025 round trips. The averaged position spread is $2.9 \mathrm{~mm}$.

In Fig. 15, the position spread of the $100^{\text {th }}$ round trip is shown together with the position spread resulting from numerical simulations. For the experimental data, the time-of-flight is normalized and the peak maximum is set to zero seconds. To obtain the position spread it is multiplied by the synchronous velocity $\left(v_{\mathrm{f}}=124.3 \mathrm{~m} / \mathrm{s}\right)$. The simulation is performed using the input parameters as discussed in Sect. 2.6. For the 


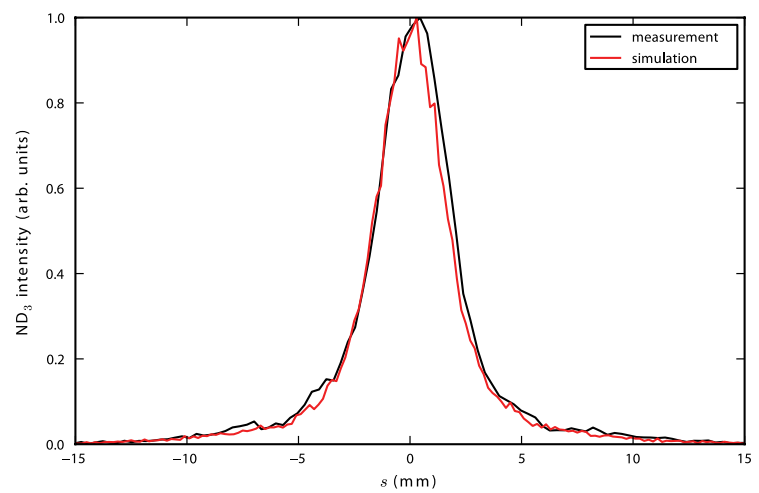

Fig. 15. The experimentally measured and simulated position spread after 100 round trips. The black curve is obtained by multiplying the measured time of flight profile shown in Fig. 14 by the velocity $(124.3 \mathrm{~m} / \mathrm{s})$ of the stored molecules. The red curve is obtained by binning the data presented in Fig. 10.

simulated data, a normalized histogram of the molecules that made 100 round trips is shown as a function of the longitudinal position $s$.

\subsection{Comparison between different bunching schemes}

The bunching scheme described in Sect. 2.5 is different from the longitudinal confinement scheme employed in the two piece molecular synchrotron. To distinguish between the two schemes the bunching of the previous molecular synchrotron is referred to as 'old scheme' and that of the current molecular synchrotron as 'new scheme'. In the old two segment molecular synchrotron, every time the molecular packet enters a gap region the electric field is switched between different configurations to achieve a longitudinal confinement force. The old bunching scheme is described in detail in references [24,27]. Briefly, when the synchronous molecule is near a gap between adjacent hexapoles, the voltages on the hexapole in which it currently resides (hexapole 1) are switched to ground while the voltages on the next hexapole (hexapole 2) are switched such as to generate a strong inhomogeneous electric field. As a result of this the molecules are decelerated when they leave the first hexapole. When the synchronous molecule is exactly between the two hexapoles, the voltages on the second hexapole are switched to ground while the voltages on the first hexapole are switched such as to generate a strong inhomogeneous electric field. As a result of this the molecules are accelerated when they enter the second hexapole. The synchronous molecule is accelerated as much as it is decelerated and its kinetic energy remains unchanged. In contrast, molecules that are in front of the synchronous molecule experience a net deceleration while molecules that are behind the synchronous molecule experience a net acceleration. The molecular packet is 'bunched' longitudinally. To compensate for the lack of transverse focusing in the gap, the confinement focusing force is increased for a short time period before and after the gap region. Compared to the bunching scheme described in Sect. 2.5, the old bunching scheme results in an effective longitudinal potential well that is ten times steeper. 

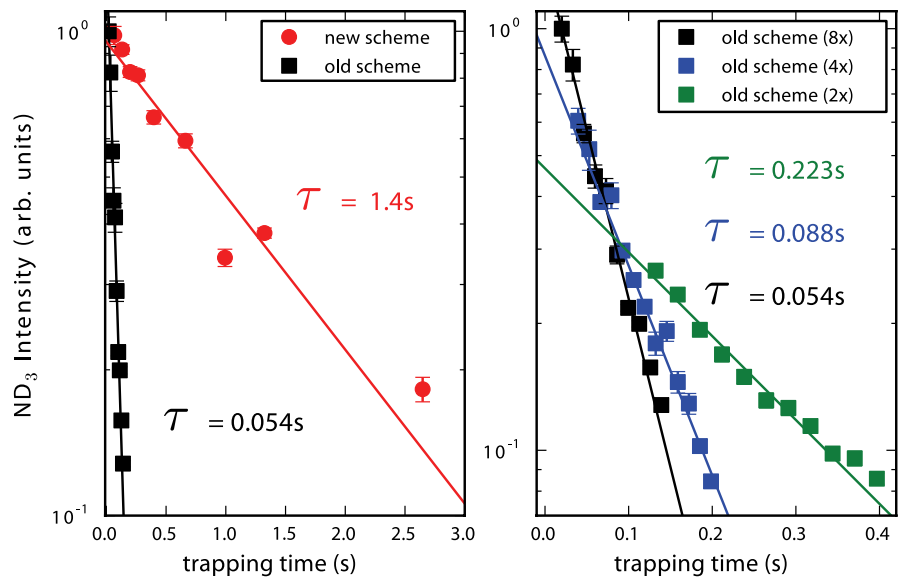

Fig. 16. Left panel: Measurements of the density of $\mathrm{ND}_{3}$. molecules as a function of time inside the 40 segment molecular synchrotron for the two different bunching schemes (logarithmic scale). For both measurements the molecular packet is bunched every fifth hexapole segment ( 8 times per round trip). The black points refer to the bunching scheme that was used in the previous molecular synchrotron consisting of two half rings ('old scheme') [24]; the red points correspond to the bunching scheme which is described in Section 2.5 ('new scheme'). The lifetime of the molecules in the synchrotron is determined from the exponential fit. Right panel: The ammonia decay measurement of the old bunching scheme for three different numbers of bunching per round trip; in black the molecular packet is bunched 8 times, in blue 4 times and in green 2 times per round trip.

To compare both switching schemes the wiring of the synchrotron is changed. Five hexapole segments are wired together (yielding 8-fold symmetry) and only one molecular packet is stored at a time. The left panel of Fig. 16 plots the density of the molecular packet for both bunching schemes on a logarithmic scale together with a fitted exponential decay. The new scheme (red circles) results in a lifetime of $1.4 \mathrm{~s}$ while the old scheme (black squares) results in a lifetime of $54 \mathrm{~ms}$. To understand the fast exponential decay, the right panel of Fig. 16 shows the exponential decay of the old scheme when the bunching procedure is applied 2 (green data points), 4 (blue data points) and 8 (black data points) times per round trip. When the number of bunching sequences per round trip is increased, the experimental decay becomes faster. The loss may be attributed to two different loss processes: (i) The molecules undergo a spin-flip transition (non-adiabatic transition) to a state that is not low-field seeking while one hexapole is grounded. (ii) Molecules are lost due to the lack of transverse focusing while the molecules are being bunched. In the experiments with the 2 segment molecular synchrotron the losses were dominated by other loss mechanisms. For the forty segment molecular synchrotron, on the other hand, the old bunching scheme leads to unacceptably large losses. The stability of the new bunching scheme well outweighs the lower acceptance of the new scheme. In addition, the new bunching scheme is less demanding for the high voltage electronics; in principle only one high voltage switch is required. The need to inject and detect multiple packets increases the number of required switches strongly (see Sect. 3.1.1). 

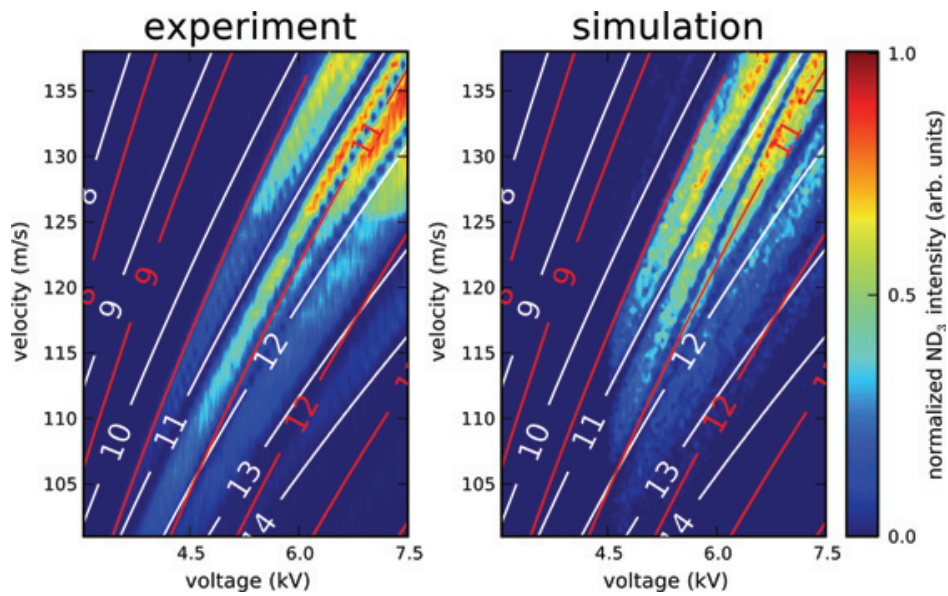

Fig. 17. Experimental and simulated ammonia intensity after 100 round trips as a function of velocity and voltage. The left panel shows the experimental data. The ammonia density is determined at 200 different voltages and 39 different velocities $(200 \times 39$ grid), averaging 52 times at each point. The right panel shows the numerically calculated intensity at 100 different velocities and 100 different voltages, resulting from simulating trajectories of 100000 molecules as they make up to 100 round trips in the synchrotron. The solid curves that overlay the contour plots indicate when the horizontal (red curve) or vertical (white curve) tune is an integer value. The tunes are calculated using Eq. (18) and the angular frequency derived from an analytic function of the electric field [38].

\subsection{Transverse motion - stopbands}

Due to imperfect alignment of the hexapoles, the confinement force varies from one segment to the next. In a ring structure this periodic variation causes motional resonances. As a result, certain forward velocities cannot be confined stably at a given hexapole voltage (see Sect. 2.4). In this section we study these so-called stopbands experimentally. Figure 17 show the ammonia intensity after 100 round trips. The left panel shows experimental data while the right panel shows the results of a numerical simulation of the experiment. The voltage is scanned between 3 and $7.5 \mathrm{kV}$ in 200 steps while the velocity is scanned between 100 and $138 \mathrm{~m} / \mathrm{s}$ in 39 steps. At each setting the signal is averaged over 52 shots. In the simulation, the trajectories of 100000 molecules are calculated at a 100 different velocities and 100 different voltages.

A number of features of Fig. 17 deserve special attention:

(i) The observed $\mathrm{ND}_{3}$ intensity is largest for high velocities simply because the beamline delivers a more intense beam (see [49]).

(ii) When the velocity is large and the voltage is relatively low (top left corner in Fig. 17) the equilibrium orbit lies outside the hexapoles and no molecules are confined.

(iii) When the velocity is small and the voltage is high (bottom right parts in Fig. 17) the observed intensity is small. Under these conditions the equilibrium radius is small and the molecules spend considerable time at the geometrical center of the hexapoles where the electric field is small. We attribute the observed losses to Majorana transitions [4,5] that transfer molecules to the untrapped $|J, M K\rangle=|1,0\rangle$ state. This is 
implemented in the simulation by assuming molecules to be lost once they experience a Stark shift below $0.015 \mathrm{~cm}^{-1}$. We chose this value to get the best agreement with the experimental data. This frequency is generated by the rapid switching (100 ns) of the electric fields.

(iv) As a consequence of (ii) and (iii), the highest intensity is observed when the velocity and voltage are such that the equilibrium radius is around $2 \mathrm{~mm}$. Note that in an ideal hexapole field when the inversion splitting is neglected, the equilibrium radius is proportional to $v_{\mathrm{f}} / \sqrt{V_{0}}$.

(v) At certain combinations of the velocity and voltage no signal is observed. These so-called 'stop bands' are the result of motional resonances when the betatron tune, the number of transverse oscillation per round trip, takes on an integer value. The white and red curves that overlay the contour plot indicate integer values for the radial or vertical betatron tune. The occurrence of stopbands implies that the alignment of the individual hexapoles is not perfect, or the voltage applied to the hexapoles is not equal for all hexapoles. In order to reproduce the measurements, we introduced random misalignments in the numerical simulations. This is done by calculating six random numbers for each segment; three of these numbers are used to shift the center of the hexapole in three directions up to $20 \mu \mathrm{m}$, while the other three are used to scale the magnitude of the force by up to $\pm 2 \%$. It is seen, that although the simulation looks very similar to the measurements, the simulated stopbands are shifted to slightly higher velocities. A possible cause for this might be that the numerically calculated electric field used in the simulations is slightly smaller than the true electric field.

From the measurements of the stopbands and with the help of Eq. (19), we can determine the horizontal and vertical betatron frequencies. From Fig. 17, we see that at a voltage difference of $6 \mathrm{kV}$, the radial and vertical tune are equal to 11 at $126.9 \mathrm{~m} / \mathrm{s}$ and $122.5 \mathrm{~m} / \mathrm{s}$, respectively. This corresponds to a radial betatron frequency of $902 \mathrm{~Hz}$ and a vertical betatron frequency of $851 \mathrm{~Hz}$. Additionally, the radial frequency can be determined when the radial tune is equal to 12 , which corresponds to a velocity of $119 \mathrm{~m} / \mathrm{s}$ at $6 \mathrm{kV}$. This second measurement of the radial frequency corresponds to $881 \mathrm{~Hz}$. The resulting average radial frequency is $891 \mathrm{~Hz}$ with an error of $11 \mathrm{~Hz}$.

\subsection{Longitudinal motion}

Non-synchronous molecules are trapped in an effective potential that moves along with the synchronous molecule. Close to the synchronous molecule the well is harmonic and non-synchronous molecules will oscillate around the synchronous molecules at the synchrotron frequency, $\omega_{\mathrm{syn}}$. In this section, we will determine the synchrotron frequency by measuring how quickly a stored packet expands if the longitudinal confinement force is switched off $-i$. e., if constant voltages are applied to the synchrotron.

The spatial distribution of the molecules as a function of time is given by

$$
\Delta s(t)=\sqrt{\left(\Delta s_{i}\right)^{2}+\left(\Delta v_{s} \cdot\left(t-t_{\min }\right)\right)^{2}} .
$$

Here, $\Delta s_{i}$ is the minimum position spread and $\Delta v_{s}$ is the velocity spread of the packet. $t_{\min }$ is the time at which the molecular packet has a minimal position spread. This time is not equal to the last time the fields are being switched but slightly later, as the molecules 

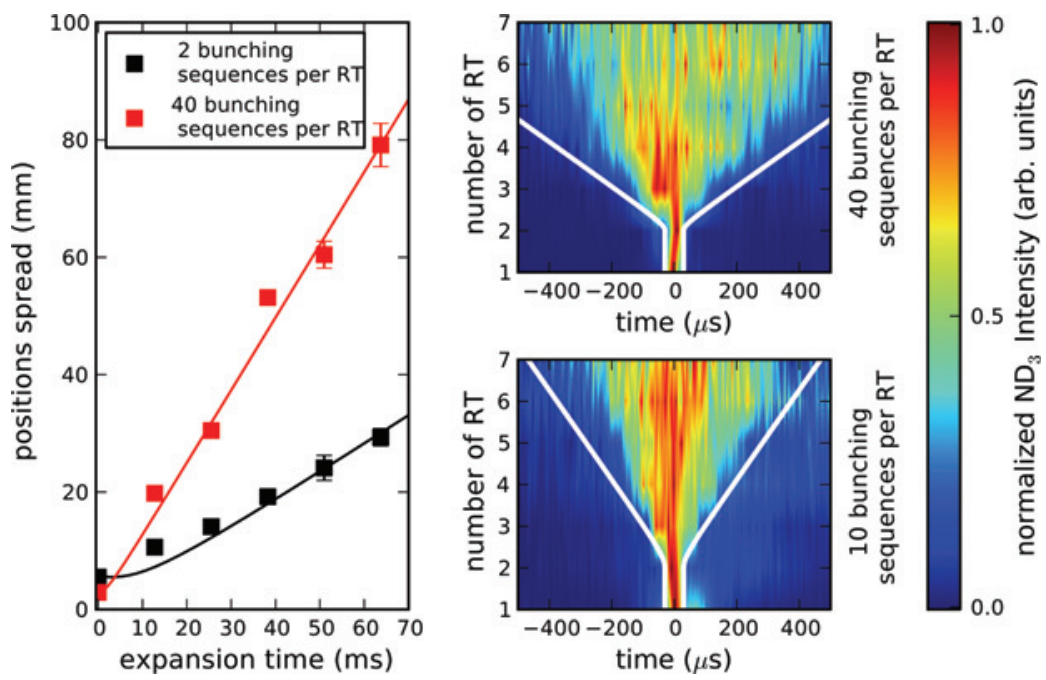

Fig. 18. The longitudinal expansion of an $\mathrm{ND}_{3}$ packet in the molecular synchrotron; the left panel shows the position spread of the molecular packet after 5 round trips (RT) as a function of the expansion time. The black and red points correspond to a situation when 2 and 40 bunching stages are used per round trip, respectively. The solid lines show the fit performed using Eq. (34). The two right panels illustrate the expansion; after the second round trip the electric field is kept constant and the packet spreads out. The upper and lower right panels show the expansion with 40 and 10 bunching sequences per round trip. The solid white line shows the theoretical expansion using Eq. (34) with $\omega_{\text {syn }} /(2 \pi)$ taking a value of 58 and $29 \mathrm{~Hz}$ for the upper and lower right panel, respectively. To achieve the best contrast for the two dimensional plots, each time-of-flight trace (horizontal axis) is normalized separately.

are first focused in free flight before they start to expand. If, e. g., the molecular packet is bunched 40 times per round trip before they are released, $t_{\min }$ is $(1 / 2) \cdot\left(L_{\mathrm{seg}} / v_{s}\right)$ after the time the fields are switched for the last time. When the molecular packet is bunched only 2 times per round trip before release, $t_{\min }$ is $10 L_{\mathrm{seg}} / v_{s}$ after the time that the fields are switched for the last time. Once the position spread and velocity spread of the packet is determined, the synchrotron frequency is found via

$$
\omega_{\mathrm{syn}}=\frac{\Delta v_{s}}{\Delta s} .
$$

The panels on the right hand side of Fig. 18 show the ammonia intensity as a function of arrival time relative to the synchronous molecule ( $x$-axis) for making a variable number of round trips after being released ( $y$-axis). The molecular packet is confined for two round trips (RT), after which the switching of the electric field is stopped. With constant voltages, the molecules are confined transversally but are free to spread out in the longitudinal direction. In the upper and lower right panels the molecular packet is bunched 40 and 10 times per round trip, respectively.

The panel on the left hand of Fig. 18 shows the longitudinal position spread of the molecular packet obtained from the time of flight data as a function of time after release. In this case the molecules have been stored for 5 round trips (RT) before release. The black and red squares correspond to measurements where the molecular 


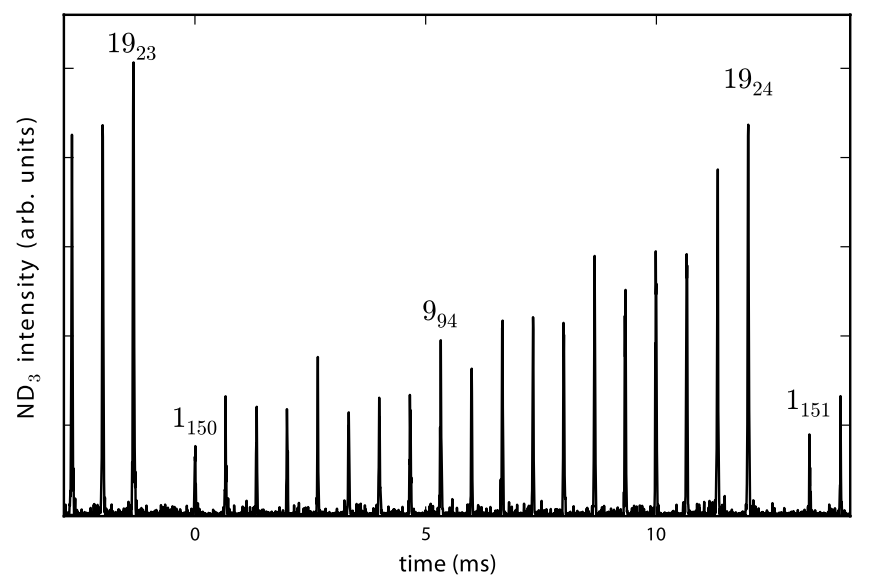

Fig. 19. Time-of-flight measurement showing 19 packets of ammonia molecules with a forward velocity of $120 \mathrm{~m} / \mathrm{s}$ revolving simultaneously in the molecular synchrotron. The horizontal axis shows the time relative to the time at which the first injected packet has completed 150 round trips. Each packet is labeled by two numbers: the main number labels the order of injection whereas the subscript labels the number of completed round trips. Figure reproduced from [28].

packet is bunched 2 and 40 times per round trip, respectively. The solid line shows the fit to the data using Eq. (34). If the packet is bunched 40 times per round trip (red curve), the fitted initial position and velocity spread are $2.9 \mathrm{~mm}$ and $1.2 \mathrm{~m} / \mathrm{s}$, respectively. Using Eq. (35), this corresponds to a synchrotron frequency, $\omega_{\mathrm{syn}} /(2 \pi)$ of $68 \mathrm{~Hz}$. As discussed in Sect. 2.5 the synchrotron frequency becomes smaller when the length between bunching stages is increased

$$
\omega_{\mathrm{syn}} \propto \frac{1}{\sqrt{L}},
$$

where $L$ is the period length. If the bunching field is switched only twice per round trip (black curve), $L$ is 20 times larger. The frequency should be a factor of 4.5 smaller, around $15 \mathrm{~Hz}$. Indeed measurements taken when the bunching field is switched twice per round trip give a position spread of $5.5 \mathrm{~mm}$ and a velocity spread of $0.5 \mathrm{~m} / \mathrm{s}$ (left panel), yielding a frequency of $14 \mathrm{~Hz}$.

\subsection{Multiple packets}

Figure 19 shows a measurement of nineteen co-propagating packets of ammonia molecules with a forward velocity of $120 \mathrm{~m} / \mathrm{s}$, loaded from beam line 1 . The horizontal axis shows the time relative to the time at which the first injected packet has completed 150 round trips. The last injected packet has been in the ring for $320 \mathrm{~ms}$ and has made 24 round trips while by then the first injected packet has already been stored for more than $2 \mathrm{~s}$. The loading scheme is such that each packet completes 7 round trips plus the length of 2 hexapoles before the next packet is injected. In the measurement the packets are seen to trail each other by $666 \mu \mathrm{s}$ - precisely the time it takes them to 
fly through two hexapoles. The time period between the first and last injected packet is equal to an effective length of four hexapole segments. The injection of multiple counter-propagating packets will be discussed in Sect. 4.

\section{Towards observing collisions}

In collision studies involving counter-propagating packets of molecules, losses in the number of stored molecules due to (in)elastic collisions accumulate over the number of interactions. Let us consider $n$ packets of molecules revolving clockwise and $n$ packets revolving anti-clockwise, all at the same speed. After $m$ round trips, each packet will have interacted $2 \cdot n \cdot m$ times with counter-propagating packets. In this section the current status of the state-of-the-art molecular synchrotron is discussed. We present experiments in which 26 packets of $\mathrm{ND}_{3}$ molecules are simultaneously confined in the synchrotron; 13 packets revolving clockwise and 13 packets revolving anti-clockwise. The sensitivity to a collision after 100 round trips will be about $10^{3}$ times increased. Unfortunately, the sensitivity of the measurements conducted has so far been insufficient to detect the small additional losses in signal brought about by collisions.

\subsection{Collisions between counter-propagating packets}

Figure 20 shows a schematic of the synchrotron that illustrates the confinement of 26 packets. The 13 orange circles indicate packets from decelerator 1 which revolve anticlockwise. The 13 packets from decelerator 2 propagate clockwise and are shown as red circles. The distance between neighboring packets is two hexapole segments while the distance between the first and the last injected packet is 16 hexapole segments. A time-of-flight measurement that corresponds to this situation is shown in Fig. 21. The molecules have a forward velocity of $124.3 \mathrm{~m} / \mathrm{s}$. The blue curve shows the ammonia intensity measured with the detector in beam line 1 , while the green curve shows the ammonia intensity measured with the detector in beam line 2. $t=0 \mathrm{~ms}$ corresponds to the time when a new packet is injected. The label above the measurements indicates the number of round trips a particular packet is confined in the synchrotron before it is detected (main number) and which beam line is used to inject it (subscript). The observed time of flight consists of a series of peaks with a periodicity of $12.7 \mathrm{~ms}$ - corresponding to the time the synchronous molecules require to complete one round trip. Let us focus on the time of flight measured with the detector in beam line 1 (blue curve). As can be seen from the labeling of the peaks, the clockwise and anti-clockwise packets partially overlap each other when being detected. The first five peaks and the last five peaks correspond to packets that are injected by beam line 1 and 2, respectively. The eight peaks in the middle of the series contain contributions from both beam lines. The time difference between adjacent peaks is $636 \mu$ s - the time it takes the molecules to pass two segments. The time difference between the last detected packet of beam line 2 and the first detected packet of beam line 1 corresponds to a spacing of 4 segments. If a new injected packet traveled ten segments in the molecular synchrotron, when it reaches the detection zone, it overlaps with the packet from the other beam line that is confined for 40 round trips. This effect can be seen around $3.2 \mathrm{~ms}$, where the $\mathrm{ND}_{3}$ intensity due to the packet from beam line 2 increases significantly. 


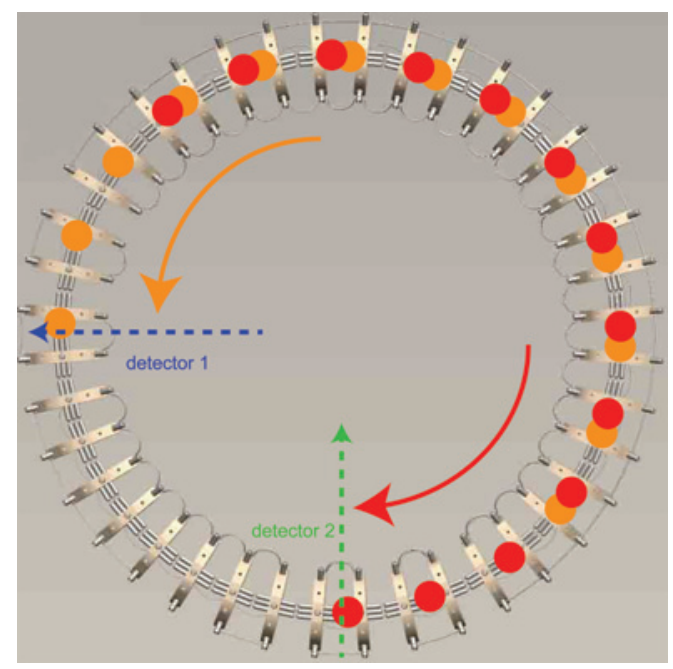

Fig. 20. A photo realistic image of the molecular synchrotron to illustrate the confinement of 26 packets. 13 packets from decelerator 1 propagate counter clockwise (orange circles) and 13 packets from decelerator 2 propagate clockwise (red circles). The distance between neighboring clockwise or anti-clockwise revolving packets is two hexapole segments. The first and the last packet are separated by 16 hexapole segments. At two points in the ring the molecular packets are both injected and detected. The dashed blue and green arrows indicate the laser beam which ionizes the molecules at the first and second detection zone, respectively.

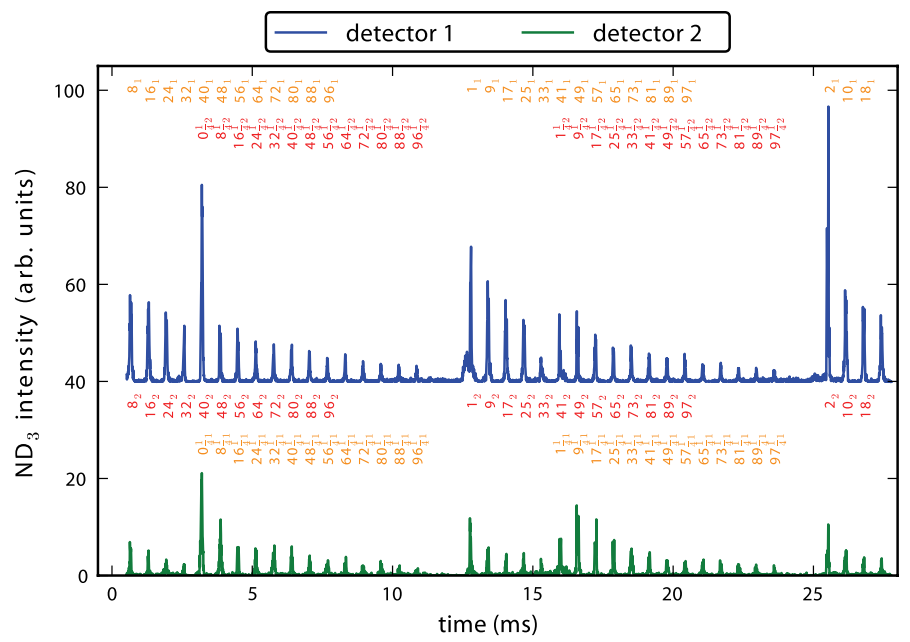

Fig. 21. A time-of-flight measurement of ammonia molecules in the molecular synchrotron when both beam lines are triggered via the continuous trigger scheme (discussed in Section 3.1.4). The signal recorded at detectors 1 and 2 is shown in blue and green, respectively. $t=0 \mathrm{~ms}$ corresponds to the time when a new molecular packet is injected. The peaks that are observed correspond to $26(2 \times 13)$ molecular packets and are labeled by two numbers. The main number indicates how many round trips the packet revolved in the molecular synchrotron before being detected; the subscript indicates the beam line that is used for its injection. The color labeling follows Fig. 20. 
Measurement scheme of a molecular collider. After successfully confining multiple counter-propagating packets, the next step is to use the molecular synchrotron as a molecular collider. We will first present a mathematical description of the experiment. Let us analyze how the ammonia intensity changes as a function of time if counterpropagating packets are present. If a molecular packet of $\mathrm{ND}_{3}$ molecules interacts with another packet of ammonia over a length of $\mathrm{d} x$, the change in the ammonia density can be expressed as

$$
\mathrm{d}\left[\mathrm{ND}_{3}\right]=-\left[\mathrm{ND}_{3}\right] \cdot \mathrm{d} x \cdot \sigma_{\mathrm{tot}} \cdot\left[\mathrm{ND}_{3}\right] .
$$

Here, we assume that the density of the two interacting packets is the same. Introducing the relative velocity

$$
v_{\text {rel }}=\frac{\mathrm{d} x}{\mathrm{~d} t}
$$

the change of the ammonia density can be rewritten as

$$
\frac{\mathrm{d}\left[\mathrm{ND}_{3}\right]}{\mathrm{d} t}=-k_{\mathrm{ND} 3}\left[\mathrm{ND}_{3}\right]^{2} .
$$

Here, $k_{\mathrm{ND} 3}$ is the decay rate due to bi-molecular collisions. Separating the variables and integrating both sides the density of the ammonia molecules as a function of time can be written as

$$
\left[\mathrm{ND}_{3}\right](t)=\frac{\left[\mathrm{ND}_{3}\right]_{0}}{1+k_{\mathrm{ND} 3} \cdot t \cdot\left[\mathrm{ND}_{3}\right]_{0}} .
$$

To understand the difficulty of colliding two Stark-decelerated molecules, let us calculate the probability of a collision taking place between an ammonia molecule with a molecule from a Stark-decelerated beam of ammonia with a density of $1 \times 10^{6}$ molecules $/ \mathrm{cm}^{3}$ and a length of $2 \mathrm{~mm}$. If we assume a total cross section for $\mathrm{ND}_{3}-\mathrm{ND}_{3}$ collisions of $500 \AA^{2}$, the probability of a collision is $P=\sigma_{\mathrm{tot}} \cdot \mathrm{d} x$. $\left[\mathrm{ND}_{3}\right]=10^{-8}$. As described above, the molecular synchrotron can enhance this signal by a factor of $10^{3}$, such that the observation of such a collision in the molecular synchrotron becomes more realistic. Because of the low probability of a collision, the expected change of the decay rate due to bi-molecular collisions in the molecular synchrotron is expected to be small. In order to detect this extra loss, the synchrotron is toggled between three different modes.

- The ammonia density is measured without counter-propagating packets (WO)

- The ammonia density is measured with counter-propagating packets (W)

- The background $\mathrm{ND}_{3}$ in the vacuum chamber is determined (BG)

The measurement without counter-propagating packets is used as a reference to include the effects of the blackbody radiation and the collisions with the background gas. The normalized collision signal is

$$
\mathrm{C}_{\text {total }}=\frac{(\mathrm{WO}-\mathrm{BG})-(\mathrm{W}-\mathrm{BG})}{(\mathrm{WO}-\mathrm{BG})} .
$$




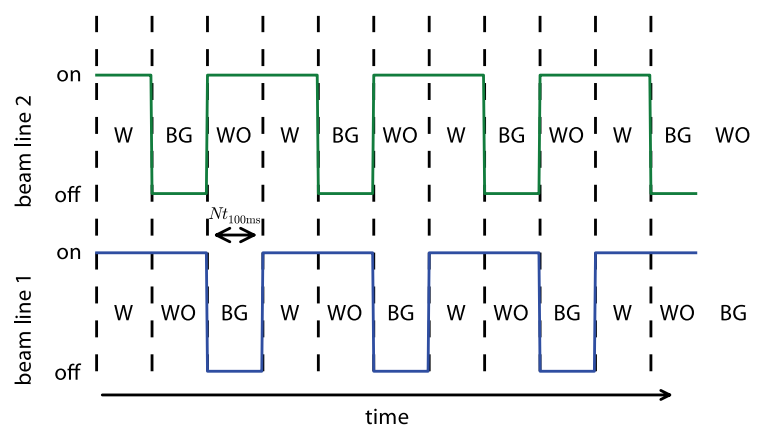

Fig. 22. The trigger scheme used for studying collisions between counter-propagating packets. To measure the collisions as sensitively as possible, the synchrotron is switched between three modes: beam lines 1 and 2 , only beam line 1 or only beam line 2 inject packets into the synchrotron. Additionally, the assignment for each beam line to the collision signal (W, WO and BG) is shown. If a molecule is detected in the W mode, counter-propagating packets are present. WO stands for 'without' counter-propagating packets. In the background measurement (BG) no molecules from this beam line are injected; only counterpropagating packets revolve in the synchrotron. The time between each mode is a integer multiple of $t_{100 \mathrm{~ms}}$.

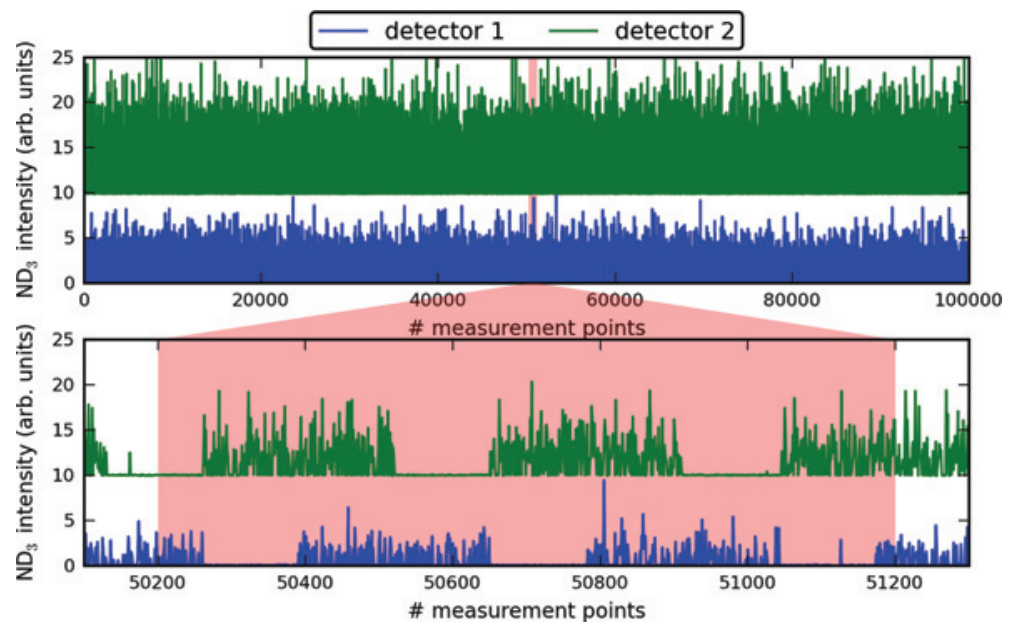

Fig. 23. An example measurement to determine collisions between counter-propagating packets of $\mathrm{ND}_{3}$ molecules. The top panel shows a 100000 single shot measurement in which a packet of molecules traveled $64 \frac{1}{4}$ round trips while the trigger scheme described in Fig. 22 is applied. The lower panel shows a zoom-in of the of the panel above. The switching trigger scheme is directly visible.

If $\mathrm{C}_{\text {total }}$ is clearly greater than zero, this is an indication that bi-molecular collisions between counter-propagating packets occur. From the increase of $\mathrm{C}_{\text {total }}$ as a function of the number of round trips, a bi-molecular collision rate can be determined.

The main advantage of this toggle mode is that slow drifts of the ammonia density, due to $e$. g. temperature changes in the valve or variation of the laser power, are compensated. The toggle scheme is sketched in Fig. 22. It shows as a function of time when the beam lines inject packets of molecules. In one mode only beam line 1 operates, in the 

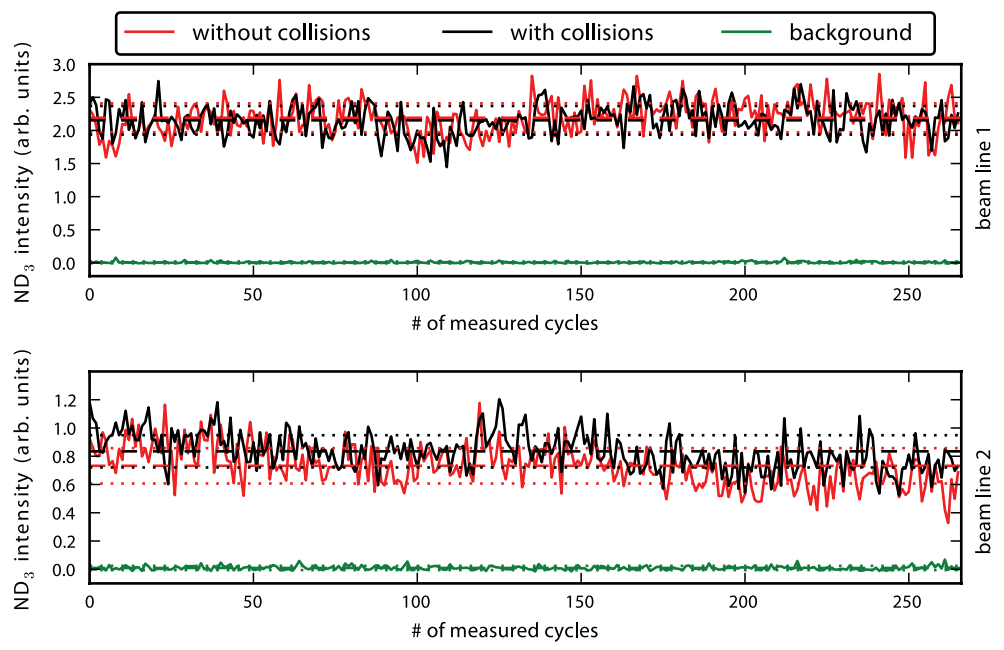

Fig. 24. The averaged $\mathrm{ND}_{3}$ signal as a function of measurement cycles using the data of Fig. 23. The top and the bottom panel show the analyzed result for beam line 1 and beam line 2, respectively. In each panel the data with collisions, without collisions and with background are shown in red, black and green, respectively. The dotted lines show the corresponding statistical error $(1 \cdot \sigma)$.

Table 2. Result of the statistical analysis of Fig. 24 for the two beam lines.

\begin{tabular}{lll}
\hline & beam line 1 & beam line 2 \\
\hline W & $2.15 \pm 0.22$ & $0.83 \pm 0.12$ \\
WO & $2.19 \pm 0.22$ & $0.73 \pm 0.13$ \\
BG & $0.01 \pm 0.02$ & $0.01 \pm 0.02$ \\
\hline
\end{tabular}

second mode only beam line 2 injects packets, while in the last mode the synchrotron confines clockwise and anti-clockwise revolving packets simultaneously. Every time the synchrotron is switched between different modes $1.3 \mathrm{~s}$ are required to load all packets. The detected molecules in this time period cannot be used for the collision analysis. To assure the continuous operation of the synchrotron the time duration of a mode is a multiple of $t_{100 \mathrm{~ms}}$. This multiple is chosen such that the ratio of the loading time and the time of one mode is minimal, while also keeping the time of one mode sufficiently short that drifts in signal level on relatively short time scales are compensated.

A first collision test. Analysis of the collision signal is only possible if the $\mathrm{ND}_{3}$ intensity is assigned correctly to one of the two injection beam lines. At certain numbers of round trips this is not possible; particularly when the counter-propagating packets overlap in the detection zone. For example the $40^{\text {th }}$ round trip cannot be used, because it overlaps with the quarter round trip from the other beam line. For this reason, only round trips 0 to 39 and $64 \frac{1}{4}$ to $103 \frac{1}{4}$ can be used for the collision analysis. In the first case the molecules are detected at the same point where they are injected. In the latter case, the detection point is a quarter round trip further than the injection point. 
Figure 23 shows a toggle measurement in which ammonia molecules are made to revolve $64 \frac{1}{4}$ round trips in the molecular synchrotron. The upper panel shows the $\mathrm{ND}_{3}$ intensity measured with detector 1 (blue curve) and detector 2 (green) for 100000 single measurements, requiring a total measurement time of $2.8 \mathrm{~h}$. The signal-to-noise ratio obtained with detector 1 is $1.2: 1$, while the one measured with detector 2 is slightly smaller. The lower panel shows an inset of the two data traces in which the toggle mode in clearly visible.

The result of the analysis of the data shown in Fig. 23 is shown in the upper and lower panel of Fig. 24 for beam line 1 and 2, respectively. The horizontal axis indicates the number of cycles while the vertical axis shows the averaged $\mathrm{ND}_{3}$ signal for the three different modes; the averaged ammonia signal with (black) and without collisions (red), and the background (green). In the analysis the first nine points of each cycle for which the loading of the molecular synchrotron is not yet completed are neglected. The total averaged signal and the corresponding variances over all cycles is shown for each trace as a dashed and dotted line, respectively. Additionally they are also listed in Table 2. As can be seen, the reduction in signal arising from bi-molecular collisions is currently smaller than the statistical error associated with the experimental measurements.

\section{Conclusion}

In this paper, we present the third generation molecular storage ring consisting of forty straight hexapoles. The new mechanical design as well as the new electronic scheme used for switching the voltages results in a very stable trap for polar molecules. The stability is illustrated by the fact that the density of the stored ammonia molecules decays at a rate of $0.31 \mathrm{~s}^{-1}$; the lowest decay rate that has been observed for neutral ammonia molecules in any trap to date. This has made it possible to observe packets of molecules after they have completed 1000 round trips in the synchrotron, thereby traveling a distance of over one mile. We present measurements that characterize the forces experienced by the molecules inside the synchrotron. A comparison between these measurements and simulations show that we have a good understanding of the synchrotron.

An important motivation of our work is the possible use of the synchrotron as a lowenergy collider for cold molecules. In Sect. 4 measurements are shown that demonstrate that 26 packets of $\mathrm{ND}_{3}$ molecules can be stored simultaneously in the synchrotron; 13 packets revolving clockwise and 13 packets revolving anti-clockwise for up to 100 round trips. This results in a $10^{3}$ fold increase in the number of collision events that take place in comparison to a crossed beam setup. Unfortunately, so far we have been unable to detect collisions between counter propagating packets. In order to be able to detect collisions in the future, we are considering possible improvements to the setup.

Every improvement that increases the lifetime of a molecular packet automatically improves the signal-to-noise ratio. One obvious way to increase the lifetime is to cool the molecular synchrotron to liquid nitrogen temperature. By this, the loss due to the blackbody radiation is reduced by a factor of 22; the decay rate of the blackbody 
process drops from $0.14 \mathrm{~s}^{-1}$ at room temperature to $0.0063 \mathrm{~s}^{-1}$ at $77 \mathrm{~K}$ [54]. A beneficial side effect is that the cooling leads to a lower pressure in the vacuum chamber. A device that shields the molecules from the blackbody radiation would at the same time function as a cold finger. Background gas will stick on the cold surface, decreasing the density of the background gas. The lifetime of the molecules confined in the synchrotron would increase significantly. Although this idea is straightforward, its implementation is not. It is mechanically and electronically highly demanding to operate a cooled molecular synchrotron. The ground potential of a cooling shield would be only a few millimeters away from electrodes or wires that are on high voltage. This may cause discharges that might make it impossible to operate the molecular synchrotron.

Another way to improve the sensitivity would be to implement velocity map imaging (VMI) [56]. With a suitable lens geometry to compensate for the astigmatic extraction fields [57], it should be possible to have a velocity resolution that is sufficient to separately detect the clockwise and anti-clockwise revolving packets. This leads to a significantly increased signal-to-noise ratio.

A completely different approach to measuring collisions would be to inject an (undecelerated) molecular beam co-propagating with the stored packets. The molecular valve that delivers the 'target' molecules can be synchronized with the cyclotron frequency, such that a stored packet of molecules will encounter a fresh target every round trip. The advantage of this method is that it offers more freedom in choosing the collision partners. For instance, in this way collisions between ammonia and hydrogen molecules or hydrogen atoms could be studied. The disadvantage is that the number of collisions now scales only linearly with the number of round trips rather than quadratically, however the greater intensity of the target beam should make up for this.

In conclusion, the molecular synchrotron presented here offers unique possibilities for collision and other types of molecular physics studies. We have completely characterized the motion of the molecules in the ring and are ready to use the synchrotron as low energy collider.

\section{References}

1. I. W. M. Smith, Low Temperatures and Cold Molecules, Imperial College Press (2008), ISBN 9781848162099.

2. R. V. Krems, B. Friedrich, and W. C. Stwalley, Cold Molecules: Theory, Experiment, Applications, CRC Press, first edition (2009), ISBN 9781420059038.

3. L. D. Carr, D. DeMille, R. V. Krems, and J. Ye, New J. Phys. 11, (2009) 055049.

4. M. Kirste, B. G. Sartakov, M. Schnell, and G. Meijer, Phys. Rev. A 79, (2009) 051401.

5. T. E Wall, S. K. Tokunaga, E. A. Hinds, and M. R. Tarbutt, Phys. Rev. A 81, (2010) 033414.

6. S. A. Meek, H. Conrad, and G. Meijer, Chem. Phys. Chem. 12, (2011) 1799.

7. D. Auerbach, E. E. A. Bromberg, and L. Wharton, J. Chem. Phys. 45, (1966) 2160.

8. J. Kalnins, G. Lambertson, and H. Gould, Rev. Sci. Instrum. 73, (2002) 2557.

9. H. L. Bethlem, M. R. Tarbutt, J. Küpper, D. Carty, K. Wohlfart, E. A. Hinds, and G. Meijer, J. Phys. B 39, (2006) R263.

10. S. Y. T. van de Meerakker, H. L. Bethlem, and G. Meijer, Nat. Phys. 4, (2008) 595.

11. S. Y. T. van de Meerakker, H.L. Bethlem, N. Vanhaecke, and G. Meijer, Chem. Rev. 112, (2012) 4828. 
12. S. Y. Lee, Accelerator Physics. 2nd edn., World Scientific, Singapore (2004), ISBN 9812562001.

13. M. Conte and W. W. MacKay, An Introduction to the Physics of Particle Accelerators. 2nd edn., World Scientific, Singapore (2008), ISBN 9812779604.

14. H. Nishimura, G. Lambertson, J. G. Kalnins, and H. Gould, Rev. Sci. Instrum. 74, (2003) 3271.

15. P. C. Zieger, A Synchrotron for Polar Molecules, Ph.D. thesis, Radboud Universiteit Nijmegen (2012).

16. H. Nishimura, G. Lambertson, J. G. Kalnins, and H. Gould, Eur. Phys. J. D 31, (2004) 359.

17. A. J. de Nijs and H. L. Bethlem, Phys. Chem. Chem. Phys. 13, (2011) 19052.

18. H. J. Loesch and B. Scheel, Phys. Rev. Lett. 85, (2000) 2709.

19. K.-J. Kügler, K. Moritz, W. Paul, and U. Trinks, Nucl. Instrum. Methods A 228, (1985) 240.

20. J. A. Sauer, M. D. Barrett, and M. S. Chapman, Phys. Rev. Lett. 87, (2001) 270401.

21. S. Wu, W. Rooijakkers, P. Striehl, and M. Prentiss, Phys. Rev. A 70, (2004) 013409.

22. A. S. Arnold, C. S. Garvie, and E. Riis, Phys. Rev. A 73, (2006) 041606.

23. F. M. H. Crompvoets, A Storage Ring For Neutral Molecules, Ph.D. thesis, Radboud Universiteit Nijmegen (2005).

24. C. E. Heiner, D. Carty, G. Meijer, and H. L. Bethlem, Nat. Phys. 3, (2007) 115.

25. D. P. Katz, J. Chem. Phys. 107, (1997) 8491.

26. F. M. H. Crompvoets, H. L. Bethlem, R. T. Jongma, and G. Meijer, Nature 411, (2001) 174.

27. C. E. Heiner, H. L. Bethlem, and G. Meijer, Chem. Phys. Lett. 473, (2009) 1.

28. P. C. Zieger, S. Y. T. van de Meerakker, C.E. Heiner, H. L. Bethlem, A. J. A. van Roij, and G. Meijer, Phys. Rev. Lett. 105, (2010) 173001.

29. N. Balakrishnan, A. Dalgarno, and R. C. Forrey, J. Chem. Phys. 113, (2000) 621.

30. D. W. Chandler, J. Chem. Phys. 132, (2010) 110901.

31. R. V. Krems, Int. Rev. Phys. Chem. 24, (2005) 99.

32. R. V. Krems, Phys. Chem. Chem. Phys. 10, (2008) 4079.

33. J. J. Gilijamse, S. Hoekstra, S. Y. T. van de Meerakker, G. C.Groenenboom, and G. Meijer, Science 313, (2006) 1617.

34. L. Scharfenberg, J. Kłos, P. J. Dagdigian, M. H. Alexander, G. Meijer, and S. Y.T. van de Meerakker, Phys. Chem. Chem. Phys. 12, (2010) 10660.

35. M. Kirste, L. Scharfenberg, J. Kłos, F. Lique, M. H. Alexander, G. Meijer, and S. Y. T. van de Meerakker, Phys. Rev. A 82, (2010) 042717.

36. M. Kirste, X. Wang, H.C. Schewe, G. Meijer, K. Liu, A. van der Avoird, L. M. Janssen, K. B. Gubbels, G. C. Groenenboom, and S. Y. T. van de Meerakker, Science 338, (2012) 1060.

37. C.E. Heiner, A Molecular Synchrotron, Ph.D. thesis, Radboud Universiteit Nijmegen (2009).

38. R. Anderson, J. Phys. Chem. A 101, (1997) 7664.

39. F. M. H. Crompvoets, H. L. Bethlem, and G. Meijer, A Storage Ring for Neutral Molecules, volume 52 of Advances In Atomic, Molecular, and Optical Physics, 209-287, Academic Press, San Diego (2005).

40. P. C. Zieger, C.E. Eyles, G. Meijer, and H. L. Bethlem, Phys. Rev. A 87, (2013) 043425.

41. G. Herzberg, Molecular Spectra and Molecular Structure: Infrared and Raman Spectra of Polyatomic Molecules, Krieger Pub Co (1991), ISBN 9780894642692.

42. Š. Urban, R. D’Cunha, K. N. Rao, and D. Papoušek, Can. J. Phys. 62, (1984) 1775.

43. L. Fusina, G. Di Lonardo, and J. W. C. Johns, J. Mol. Spectrosc. 118, (1986) 397.

44. K. Shimoda, Y. Ueda, and J. Iwahori, Appl. Phys. A 21, (1980) 181.

45. S. R. Gandhi and R. B. Bernstein, J. Chem. Phys. 87, (1987) 6457.

46. H. L. Bethlem, F. M. H. Crompvoets, R. T. Jongma, S. Y.T. van de Meerakker, and G. Meijer, Phys. Rev. A 65, (2002) 053416.

47. D. A. Dahl, Simion 3D Version 8.0 (1995).

48. C.E. Heiner, G. Meijer, and H. L. Bethlem, Phys. Rev. A 78, (2008) 030702.

49. C. E. Heiner, H. L. Bethlem, and G. Meijer, Phys. Chem. Chem. Phys. 8, (2006) 2666.

50. M. N. R. Ashfold, R. N. Dixon, N. Little, R. J. Stickland, and C. M. Western, J. Chem. Phys. 89, (1988) 1754. 
51. W. C. Wiley and I. H. McLaren, Rev. Sci. Instrum. 26, (1955) 1150.

52. Spectra-Physics, Quanta-Ray PRO-Series Pulsed Nd:YAG Lasers (2002).

53. Behlke Power Electronics GmbH, Fast High Voltage Transistor Switches HTS-GSM (2010).

54. S. Hoekstra, J. J. Gilijamse, B. Sartakov, N. Vanhaecke, L. Scharfenberg, S. Y. T. van de Meerakker, and G. Meijer, Phys. Rev. Lett. 98, (2007) 133001.

55. J. van Veldhoven, R. T. Jongma, B. Sartakov, W. A. Bongers, and G. Meijer, Phys. Rev. A 66, (2002) 032501.

56. A. T. J. B. Eppink and D. H. Parker, Rev. Sci. Instrum. 68, (1997) 3477.

57. M. Quintero-Pérez, P. Jansen, and H. L. Bethlem, Phys. Chem. Chem. Phys. 14, (2012) 9630. 